\title{
ETHNOMEDICINAL PLANTS USED AGAINST COMMON DIGESTIVE PROBLEMS
}

\author{
Muhammad Adnan $^{1 *}$, Roqaia Bibi ${ }^{1}$, Azizullah Azizullah ${ }^{1}$, Rani Andaleeb ${ }^{2}$, Sakina Mussarat ${ }^{1}$, Akash \\ Tariq $^{1}$, Riaz Ullah ${ }^{3}$, Naser M. AbdElsalam ${ }^{4}$, Abdul Latif Khan ${ }^{5}$, Shaheen Begum ${ }^{6}$ \\ ${ }^{1}$ Department of Botany, Kohat University of Science and Technology, Kohat-26000, Pakistan \\ ${ }^{2}$ Department of Microbiology, Kohat University of Science and Technology, Kohat-26000, Pakistan \\ ${ }^{3}$ Department of Chemistry, Government College Ara Khel, Frontier Region Kohat-26000, Pakistan \\ ${ }^{4}$ Riyadh Community College, King Saud University, Riyadh-11437, Saudi Arabia, ${ }^{5}$ UoN Chair of Oman's \\ Medicinal Plants \& Marine Natural Products, University of Nizwa, Nizwa, Oman, ${ }^{6}$ Department of \\ Environmental Sciences, Fatima JinnahWomen University, The Mall Rawalpindi-46000, Pakistan. \\ "E-mail: ghurzang@ hotmail.com
}

\begin{abstract}
Background: Ethnomedicinal knowledge is highly significant for persistence of human health care. Different studies have shown that medicinal plants are considered as curing agent for digestive disorders in Pakistan. The aim of present review is to explore this ethnomedicinal information all over the Pakistan and to investigate the ecological status of these species in the country.

Methodology: Online literatures on ethnomedicinal plants used to treat digestive complaints in Pakistan were searched and gathered using online bibliographic databases including PubMed, Scopus, Google Scholar, Web of Science and Flora of Pakistan.

Results: The review documented 178 medicinal plant species, belonging to 59 families, used for different digestive ailments. The majority of species were herbs $(60 \%)$, used in drug preparation. The most frequent part used in preparation of these remedies is leaves $(23 \%)$, followed by roots $(19 \%)$.

Conclusion: In conclusion, the study provides enormous ethno-medicinal knowledge and due to the unsustainable activities of the people of Pakistan, some medicinal plants need to be conserved for the future.
\end{abstract}

Key words: Ethno-medicine, medicinal plants, gastrointestinal diseases, ecological status.

\section{Introduction}

Plants are a vital source of traditional medicines that are used for the treatment of various ailments. Approximately 4, 22,000 flowering plants reported from the world, more than 50,000 have been used worldwide for medicinal purposes (Walter \& Hamilton, 1993). Man has cultivated the habit of observing plants for thousands of years and had used them for different purposes (Bako et al., 2005). The local uses of medicinal plants are common particularly in those areas of developing countries, which have little or no access to modern health services (Sandhya et al., 2006). Mostly local people keeping the centuries-old trend alive, rely hugely on the wild plants for their daily needs such as food, fodder and medicines. Treatment through traditional medicine system is progressing all over the world particularly in the Indo-Pak sub-contentment where this system is known by Unani or Ayurvedic (Haq, 1993).

Pakistan has a unique biodiversity that is stretched along nine major ecological zones. A major part of the country is quite rich in medicinal herbs due to its salubrious climate (Abbasi et al., 2010). Numerous medicinal plants are being used by local communities of different geographical regions of the country, having old traditional knowledge on such plants. Medicinal plants have been used as a source of curing various digestive disorders in the country. Digestive disorders generally occur due to the nature of food that we consume and it's incompatibility with our constitution or due to some viruses and bacteria like rotavirus, Helicobacter pylori, Salmonella, Shigella and Escherichia coli etc. Some of the most common digestive problems that people of Pakistan faces in their day to day life are stomach-aches, cramps, vomiting, dyspepsia, diarrhea and indigestion to the complicated conditions such as cancer and gastric ulcer etc (Zaidi et al., 2009, Khan et al., 2013). In early 1950, more than $80 \%$ of Pakistani population was totally dependent on ethnomedicines for traditional health practices (Hocking, 1958), but now, it is experienced only in the rural areas (Ibrar et al., 2007), because the indigenous knowledge changes with the passage of time, with change of natural resources and culture. Indigenous knowledge on ethnomedicines is under threat due to the current modernizing trends. In Pakistan, about 6000 plant species have been reported so far. However, only 600 plant species have been documented recently (Shinwari et al., 2003). Therefore, there is a dire need to preserve this valuable traditional knowledge (Khan et al., 2011) by conserving plants and educating people about ethnomedicinal knowledge. Conservation of medicinal plants is a serious issue of present times. Because studies have shown that plant biodiversity of Pakistan is under wonderful pressure due to the population explosion and unsustainable activities of people in the country (Abbas et al., 2010, Sheikh et al., 2002).Unfortunately, very little work has been done on threatened plants of the country and extremely limited information is available on this subject (Alam \& ali., 2009). Thus the present review aims to collect and elaborate the ethnomedicinal data available for digestive problems in Pakistan. This could help in creating mass awareness regarding the need for conservation of endangered, threatened and vulnerable medicinal plant species used ethnobotanically in the country. The review also helps in the promotion of ethno-medico-botany knowledge all over the world. Hence people belonging to the countries having these species may also use the remedies mentioned in this paper for the treatment of their digestive problems. Above all, this review will provide baseline information for ecologists, pharmacists and researchers for further studies.

\section{Methodology}

This review paper was designed by consulting and compiling large number of mostly published literature on gastrointestinal 
Adnan et al., Afr J Tradit Complement Altern Med. (2015) 12(5):99-117

\section{http://dx.doi.org/10.4314/ajtcam.v12i5.15}

problems of Pakistan. Literature on ethnobotanical information of medicinal plants against gastrointestinal diseases in the country has been searched using online bibliographic databases including PubMed, Scopus, Google Scholar, Web of Science and Flora of Pakistan. An extensive number of articles were found out of which approximately 105 articles were selected as the data was focused to only those medicinal plants whose recipes were mentioned for the gastrointestinal ailments. The material was thoroughly checked for the exclusion of duplicate material collected from different search engines. A detailed ethnomedicinal table was formulated on all the plants used for gastrointestinal complications in a systematic manner. In ethnomedicinal table, plant scientific names, local names, habit, reproductive status, part used, recipes and types of problems were included. Efforts were also made, to collect data regarding ecological status of these species in the country. However, this data was found limited and was compiled in table form.

\section{General Overview}

Traditionally, different medicinal plants are used for various gastrointestinal problems such as diarrhea, dyspepsia, peptic ulcer vomiting and nausea etc. in the developing countries like Pakistan. In present review, a total of 178 plants belonging to 122 genera and 59 families are documented to be used in different regions of the country. These plants include Achillea millefolium, Achillea wilhelmsii, Achyranthes aspera, Adhatoda vasica, Allium sativum and many others (Table 1). Literature proved that herbs were mostly used $(60 \%)$ in various gastrointestinal related ethnomedicinal recepies followed by shrub (21\%) (Figure 1.) It might be due to the fact that herbs can be easily uprooted and extracted from the wild or due to their strong therapeutic activity (Murad et al., 2013). Most of the plants (50\%) are annuals followed by perennials plants (44\%) and 6\% biennials. Different plant parts are used for remedy preparation in order to treat digestive disorders but leaves are mostly preferred (23\%) followed by roots (19\%), pulp (15\%), whole plant (12\%), fruits (11\%), stem (9\%) and 5\% bark. However, other parts of the plants such as latex, rhizome and resin etc., are also used in some of herbal preparation. The use of specific plant parts for specific disease treatment suggests that these parts have strongest certain active phyto-constituents against digestive complaints with no or less side effects (Mitscher et al., 1980). Moreover, the maximum use of leaves for remedy preparation might be due to the reason that it is main site of most of the physiological process that result in production of variety of active compounds as well as the collection of leaves poses no significant threat to the survival of individual plants (Poffenberger et al., 1992). This article critically reviews only some common digestive disorders and their phytotherapy with locally used medicinal plants; furthermore, the ecological status of these species in the country is also discussed. Out of 178 species, the ecological status of only 34 plants was discussed in literature (Table 2). It was noticed that 14 plants were endangered and needs to be conserved otherwise they will be extinct in future. Similarly, 6 medicinal plants were found threatened and are at risk of becoming endangered in future if not protected (Figure 2).

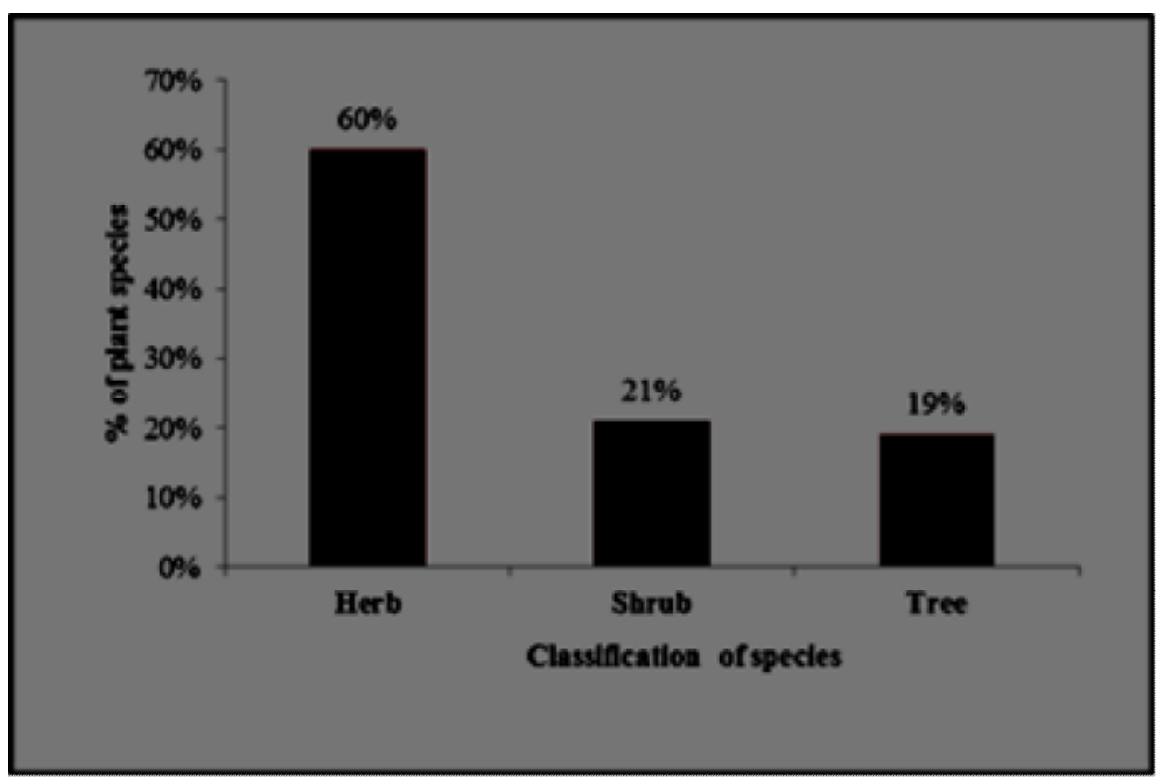

Figure 1: Classification of plants on the basis of their habits. 


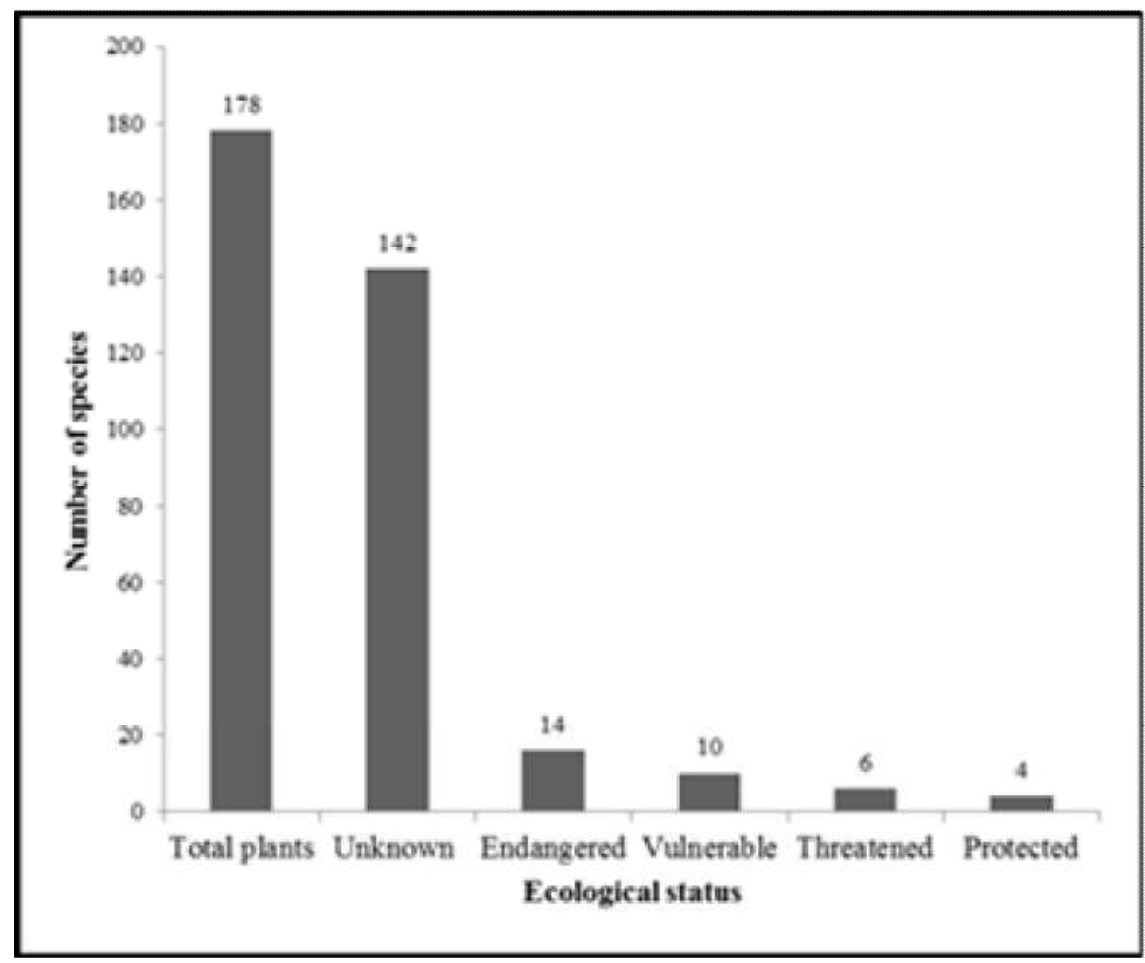

Figure 2: Ecological status of plants used for digestive problems in Pakistan

\section{Diarrhea and Dysentery}

Diarrhea and dysentery are most commonly occurring digestive disorders. Dysentery is the severe form of diarrhea which contains blood. Diarrhea is caused by rotavirus, adenovirus and or various bacterial species belonging to the Aeromonas, Cryptosporidium, Campylobacte, Salmonella, Shigella, Escherichia coli etc. (Shinwari et al., 2013, O'Ryan et al., 2005). In the developing countries like Pakistan, diarrhea is the third leading cause of death (Thapar \& Sanderson, 2004). People with poor hygiene, children and adults are at high risk. More than 3.2 million children of developing countries died annually because of diarrhea (Rebiero, 2000). In Pakistan, different medicinal plants such as Acorus calamus, Albizia lebbeck, Allium sativum, Amaranthus viridis, Foeniculum vulgare, Mentha spicata and Adhatoda vesica etc., are used in order to cure the ailments. The decoction method is mostly preferred ethnobotanically for remedy preparation in the country. Traditionally, the bark of Albizia lebbeck in addition to Trchyspermum ammi and Commelina bengalensis are boiled in water to prepare a decoction for the diseases of diarrhea and dysentery. Moreover, people of Pakistan boil leaves, fruit and stem of Salvadora oleoides in water and locally the decoction is given to patients suffering from diarrhea (Table 1). However, due to human unsustainable activities, these plants are endangered in Pakistan (Table 2). If these are used with maximum ratio, they might become extinct in future. Similarly, some other anti-diarrheal and anti-dysenteric plants of Pakistan are traditionally administered in different ways, such as roots and leaves of Barberis lyceum are boiled and decoction is used against the diseases, in addition the roots of Bergenia ciliate are used as a tonic in the treatment of diarrhea (Table 1). However, these are considered as threatened species of the country and might be endangered in future, if not protected. In addition, researchers scientifically proved some medicinal plants of Pakistan as vulnerable species which are used against diarrhea. Examples are Achillea millefolium, Elaeagnus angustifolia and Ocimum bacilum (Table 2). These vulnerable plants are administered for the disease treatment. Literally, there is need to conserve all the above species and instead of them being protected or conserved medicinal plants should be preferred against diarrhea and dysentery. For example Allium sativum is protected plant species of Pakistan (Table 2) and should be preferred for the disease curing. This review calls for an urgent complementary conservation action to save these antidiarrheal and anti-dysenteric medicinal plant species in Pakistan and clinical studies are also required to justify the ethnomedicinal knowledge.

\section{Peptic Ulcer}

Peptic ulcer, being an inflamed break in the mucosa skin, can be divided into two broad categories that include ulcer in the stomach (gastric) and the duodenum (Tarnawski, 2005, Gadekar et al., 2010). The prevalence of gastrointestinal ulcers differs around the world: in Asia gastric ulcer is dominant while duodenal ulcer is more frequent in Western populations of the world (Yuan et al., 2006). The etiological factors of this disorder include: exogenous aggressive factors such as stress, smoking, nutritional deficiencies, infections due to Helicobacter pylori, frequent and indiscriminate use of non-steroidal anti-inflammatory drugs (Khazaei et al., 2006, Bandyopadhyay et al., 2001). During the past few decades, researchers were searching to identify new anti-ulcer therapies from natural sources. Thus herbs, spices, vegetables and crude drug substances are considered to be a potential source to treat these ailments and 


\section{Adnan et al., Afr J Tradit Complement Altern Med. (2015) 12(5):99-117 http://dx.doi.org/10.4314/ajtcam.v12i5.15}

scientific literature, also reported a large number of medicinal plants with anti-ulcer potential (Al-Mofleh et al., 2006, Raffatullah et al., 1990, Al-Mofleh et al., 2008). In Pakistan different medicinal plants are used traditionally for the treatment of peptic ulceration. For example Achyranthes aspera is a perennial wild endemic herb of Pakistan (Table 2). It is boiled in water and the decoction is used for the treatment of ulcer. Similarly, decoctions of other medicinal plants are also recommended for the disease treatment such as seeds of Cordia oblique and Fruit, bark of Capparis deciduas are boiled, in order to make decoctions for the phytotherapy. The decoction of C. oblique is administered orally for 10-15 days to the patients. Similarly, the decoction and infusion are prepared from the leaves of Oxystelma esculentum and recommended as mouth wash for the treatment of ulceration in Pakistan. Leaves of Alstonia scholaris are pulverised to make poultice for peptic ulcer. Furthermore, the powdered flowers of Rosa damascena are mixed with with Quercus fruits and an infusion is prepared to be used against stomach ulcer (Table 1). From literature, it is noticed that there is no available data regarding the ecological status of the above anti-ulcer plants. Thus, further studies are required to gather ecological information in order to conserve these species if they are endangered or threatened.

\section{Oropharyngeal and Gastric Cancer}

According to World Gastrointestinal Organization, it is noticed that sometimes the malignant lesions occur in the oropharyngeal wall and overall, 15 to $20 \%$ of these lesions progress to carcinoma (Lambert, 2010). However, cancer is not only limited to the pharyngeal region, it also affects other parts of digestive system like stomach and known as gastric cancer. This disease is mostly found in young population. According to a study it was noticed that the existence of malignant gastrointestinal tumors is found in higher frequency in the young population of Pakistan (Butt et al., 2012). The causal factors of cancer include tobacco smoking or chewing, alcohol drinking, socioeconomic status and nutritional deficiencies, Human Papilloma Virus and Helicobacter pylori (Lambert, 2010).

This review notifies that there must be some anti-cancerous medicinal plants in the country but they have no ethnobotanical and pharmacological proves. Therefore, they should be checked for gastrointestinal cancer in Pakistan.

\section{Dyspepsia}

Dyspepsia is defined as epigastric fullness after meals, often described by the patients as indigestion. It affects 14 to $40 \%$ population of world annually. The symptoms of dyspepsia usually contain upper abdominal fullness, eructation, heartburn, nausea and vomiting (Zaidi et al., 2009; Tally et al., 1999). In Pakistan, different medicinal plants are recommended for the treatment of dyspepsia. Mostly decoction and powdered form of the plants are used for remedy preparation in the country. Foeniculum vulgare is a perennial herb in Pakistan; its fruits are directed for the treatment of dyspepsia. Traditionally, 250gm of dried fruits is grinded to obtain powder. This powder is mixed with $250 \mathrm{gm}$ seeds of pomegranate and $375 \mathrm{gm}$ of sugar candy, $4-6$ seeds of Green cardamom in powder form are added. One teaspoon of this powder is used thrice a day before and after meal, in order to cure the dyspepsia (Table 1). According to Zaidi et al., green cardamom is employed traditionally more for dyspeptic symptoms than black cardamom. As Helicobacter pylori is considered to be one of the causative factors for dyspepsia, the comparatively strong bactericidal activity of green cardamom might explain its traditional use (Zaidi et al., 2009). In addition, some other plant species such as Zanthoxylum armatum, Elaeagnus angustifolia and Echinops echinatus are also used for the dyspepsia treatment. However, due to unsustainable activities of people, some species are at risk of becoming extinct in future, if not protected. For example Z. armatum wild endemic shrub of Pakistan is critically endangered (Table 2) and needs to be conserved. This species is traditionally recommended in the treatment of dyspepsia or its symptoms and 50gm seeds or fruits of the plant are mixed with flour and is orally given to patients. Similarly, E. angustifolia is vulnerable perennial tree in the country. Its fruit Juice, seeds and roots is recommended traditionally against gastrointestinal ailment (Table 1). Being the vulnerable species it also needs to be conserved for the future. The fruit extract of Olea ferrugini is given orally for 5-6 days to dyspepsia patients. This medicinal plant is also endangered in Pakistan (Table 2). Therefore, it is necessary that people of the country should use protected plant species in place of endangered, threatened and/or vulnerable species. Additionally, further studies are required to find out the ecological status of species in the country because limited literature is available.

\section{Conclusions}

The study provides enormous ethno-medicinal knowledge all over the world administered via different recipes for digestive problems. However, the phytoterapy should be checked for gastrointestinal cancer in Pakistan. Further, due to unsustainable activities of people of Pakistan, some medicinal plants like Acacia nilotica, Acorus calamus, Aesculus indica, Albizia lebbeck, and Zanthoxylum armatum etc. are at risk of becoming extinct in future, so it is a dire need to conserve these species. Additionally, the ecological status of most species is unknown, so these findings are research provoking for future. Furthermore, clinical studies are required to scientifically justify the rationale of traditional uses of medicinal plants of Pakistan.

\section{Acknowledgements}

The authors are thankful to the Deanship of Scientific Research, King Saud University, Riyadh, Saudi Arabia, for funding the work through the Research Group Project no. RGP-210. The authors are highly indebted to departmental colleagues for their support. 
Adnan et al., Afr J Tradit Complement Altern Med. (2015) 12(5):99-117

http://dx.doi.org/10.4314/ajtcam.v12i5.15

\begin{tabular}{|c|c|c|c|c|c|c|c|c|}
\hline Plant species/ Families & $\begin{array}{l}\text { Local } \\
\text { name }\end{array}$ & $\begin{array}{l}\text { Plant } \\
\text { status }\end{array}$ & Habit & $\begin{array}{l}\text { Part } \\
\text { used }\end{array}$ & ( & Diseases & Place & Refrences \\
\hline $\begin{array}{l}\text { Abrus precatorius } \mathrm{L} \text {. } \\
\text { Papilionaceae }\end{array}$ & Rati & $\mathrm{P}$ & $\mathrm{T}$ & $\mathrm{L}$ & $\begin{array}{l}\text { Decoction having little common salt is given twice a day for } \\
2 \text { days }\end{array}$ & Stomach pain & $\begin{array}{l}\text { Lahore-Islamabad } \\
\text { motorway }\end{array}$ & Rashid., 2012 \\
\hline $\begin{array}{l}\text { Abutilon indicum } \mathrm{L} . \\
\text { Malvaceae }\end{array}$ & $\begin{array}{l}\text { Sumbal } \\
\text { Kakpai }\end{array}$ & $\mathrm{P}$ & $\mathrm{H}$ & WP & Juice of plant and decoction is used orally & Dysentery & $\begin{array}{l}\text { Bannu, Dara Adam } \\
\text { khel }\end{array}$ & $\begin{array}{l}\text { Marwat et al.,2012; Mahmood } \\
\text { et al.,2013 }\end{array}$ \\
\hline $\begin{array}{l}\text { Acacia catechu } \\
\text { L.Mimosaceae }\end{array}$ & Kikar & A & $\mathrm{T}$ & $\begin{array}{l}\text { W } \\
\text { Sp }\end{array}$ & $\begin{array}{l}\text { Decoction of wood. } 150 \mathrm{~g} \text { of dried sap is mixed with } 300 \mathrm{ml} \\
\text { water and given } 1 \mathrm{~h} \text { intervals for } 24 \mathrm{~h} \text {. }\end{array}$ & $\begin{array}{l}\text { Intestinal pain } \\
\text { Blood dysentery }\end{array}$ & $\begin{array}{l}\text { Northern Areas of } \\
\text { Pak }\end{array}$ & $\begin{array}{l}\text { Singh et al.,2012; } \\
\text { Rahmattullah et al.,2010 }\end{array}$ \\
\hline $\begin{array}{l}\text { Acacia modesta Wall. } \\
\text { Mimosaceae }\end{array}$ & Palosa & $\mathrm{P}$ & $\mathrm{H}$ & B & $\begin{array}{l}\text { Bark powder of bark mixed with little quantity of salt and } \\
\text { sugar and used }\end{array}$ & $\begin{array}{l}\text { Stomachache } \\
\text { Dysentry }\end{array}$ & $\begin{array}{l}\text { Northern Areas of } \\
\text { Pak }\end{array}$ & $\begin{array}{l}\text { Sher et al.,2011; Hayat et } \\
\text { al.,2007 }\end{array}$ \\
\hline $\begin{array}{l}\text { Acacia nilotica } \mathrm{L} . \\
\text { Mimosaceae }\end{array}$ & Kikar & $\mathrm{A}$ & $\mathrm{T}$ & $\begin{array}{l}\text { B } \\
\text { S }\end{array}$ & $\begin{array}{l}\text { Decoction of } 1 / 2 \mathrm{~kg} \text { bark is given orally twice a day for 5-6 } \\
\text { days. Shoots powder along with zeera and pomegranate } \\
\text { flowers use orally as carminative. }\end{array}$ & $\begin{array}{l}\text { Stomach disorder } \\
\text { Carminative }\end{array}$ & Southern Punjab & $\begin{array}{l}\text { Singh et al.,2012; Khan et } \\
\text { al.,2013; Abbas et al.,2013 }\end{array}$ \\
\hline $\begin{array}{l}\text { Acacia Senegal L. } \\
\text { Mimosaceae }\end{array}$ & Kikar & A & $\mathrm{T}$ & G & Fresh gum is eaten & $\begin{array}{l}\text { Constipation, } \\
\text { Stomachache }\end{array}$ & Southern Punjab & $\begin{array}{l}\text { Khan et al.,2013; Belayneh et } \\
\text { al.,2012 }\end{array}$ \\
\hline $\begin{array}{l}\text { Achillea millefolium } \mathrm{L} \text {. } \\
\text { Asteraceae }\end{array}$ & $\begin{array}{l}\text { Sultani } \\
\text { Booti }\end{array}$ & A & $\mathrm{H}$ & WP & $\begin{array}{l}\text { Decoction of flower in milk is laxative. Tea made from the } \\
\text { plant is used for dirrhoea. Whole plant extract is used in } \\
\text { constipation. Fresh or dried ground leaves for relieving } \\
\text { anorexia, constipation andstomachache. }\end{array}$ & $\begin{array}{l}\text { Laxative, Anorexia } \\
\text { Constipation, Dirrhoea } \\
\text { Constipation } \\
\text { Stomachache }\end{array}$ & $\begin{array}{l}\text { Khirthar National } \\
\text { Park }\end{array}$ & $\begin{array}{l}\text { Awan et al.,2013; Ballabh \& } \\
\text { Chaurasia., 2009 }\end{array}$ \\
\hline $\begin{array}{l}\text { Achillea wilhelmsii } \\
\text { C.Koch. } \\
\text { Asteraceae }\end{array}$ & $\begin{array}{l}\text { Boi baro, } \\
\text { Gozh } \\
\text { madar }\end{array}$ & $\mathrm{P}$ & $\mathrm{H}$ & WP & Decoction is used & $\begin{array}{l}\text { Dirrhoea } \\
\text { Constipation } \\
\text { Stoachache }\end{array}$ & $\begin{array}{l}\text { Khirthar National } \\
\text { Park }\end{array}$ & $\begin{array}{l}\text { Ali \& Qaiser., 2009; Tareen et } \\
\text { al.,2010 }\end{array}$ \\
\hline $\begin{array}{l}\text { Achyranthes aspera } \mathrm{L} \text {. } \\
\text { Amaranthaceae }\end{array}$ & $\begin{array}{l}\text { Kandri, } \\
\text { Puth Kanda }\end{array}$ & $\mathrm{P}$ & $\mathrm{H}$ & WP & $\begin{array}{l}\text { Extract is used for different gastro complaints. Seeds } \\
\text { powder mixed with sugar and given in colic.Root paste } \\
\text { mixed with black pepper is taken during cholera, indigestion }\end{array}$ & $\begin{array}{l}\text { Dysentery, Ulcer } \\
\text { Abdominal pain, } \\
\text { Colic,Cholera, } \\
\text { Indigestion }\end{array}$ & $\begin{array}{l}\text { Nwazstan } \\
\text { ANP }\end{array}$ & $\begin{array}{l}\text { Arshad et al.,2014; Qureshi et } \\
\text { al.,2011; Shadangi et al.,2012 }\end{array}$ \\
\hline $\begin{array}{l}\text { Acorus calamus } \mathrm{L} . \\
\text { Acoraceae }\end{array}$ & Kini kathi & $\mathrm{B}$ & $\mathrm{H}$ & $\mathrm{Rh}$ & Powder is given & Dysentery, Diarrhea & Cholistan & Hamayun et al.,2006 \\
\hline $\begin{array}{l}\text { Acroptilon repens } \mathrm{L} . \\
\text { Asteraceae }\end{array}$ & Talkha & $\mathrm{P}$ & $\mathrm{S}$ & WP & $\begin{array}{l}\text { Grinded leaves are mixed with flour and water to make } \\
\text { paste and applied externally on belly }\end{array}$ & $\begin{array}{l}\text { Stomachache } \\
\text { Dysentery }\end{array}$ & $\begin{array}{l}\text { Lahore-islamabad } \\
\text { motoway }\end{array}$ & Ali \& Qaiser., 2009 \\
\hline $\begin{array}{l}\text { Adhatoda vasica Nees. } \\
\text { Acanthaceae }\end{array}$ & Bahker, & $\mathrm{P}$ & $\mathrm{H}$ & $\mathrm{L}$ & $\begin{array}{l}1 \mathrm{~kg} \text { fresh leaves each of } A . \text { vasica and Rhazya stricta are } \\
\text { soaked in water over night and administered for 3-4 days. }\end{array}$ & Stomach disorder & Cholistan & Abbas et al.,2013 \\
\hline $\begin{array}{l}\text { Adhatoda zeylanica } \\
\text { Medic.Acanthaceae }\end{array}$ & Bhaikar, & $\mathrm{P}$ & $\mathrm{H}$ & WP & Root and leaves decoction is given & Anthelmentic & Cholistan & $\begin{array}{l}\text { Rauf et al.,2012; Khan \& } \\
\text { Hanif., } 2006\end{array}$ \\
\hline $\begin{array}{l}\text { Aesculus indica } \text { Coleb. } \\
\text { Hippocastanaceae }\end{array}$ & Jawaz & $\mathrm{A}$ & $\mathrm{T}$ & $\mathrm{F}$ & Powder is mixed with husk and taken & Abdominal pain, Colic & $\begin{array}{l}\text { Jalalpur Jattan, } \\
\text { Gujrat }\end{array}$ & $\begin{array}{l}\text { Abbas et al.,2013; Ali \& } \\
\text { Qaiser., 2009 }\end{array}$ \\
\hline $\begin{array}{l}\text { Agave americana } \mathrm{L} . \\
\text { Agavaceae }\end{array}$ & $\begin{array}{l}\text { Kanwar } \\
\text { Phara }\end{array}$ & $\mathrm{A}$ & $\mathrm{T}$ & $\mathrm{P}$ & Make Halwa and used & Constipation, Acidity & Buner & Qureshi et al.,2011 \\
\hline $\begin{array}{l}\text { Agave sisalana Perr. } \\
\text { Agavaceae }\end{array}$ & $\begin{array}{l}\text { Kanwar } \\
\text { Phara }\end{array}$ & $\mathrm{P}$ & $\mathrm{T}$ & $\mathrm{P}$ & Make Halwa and used for constipation and acidity & $\begin{array}{l}\text { Constipation, Piles, } \\
\text { Acidity }\end{array}$ & Cholistan & Manan et al.,2007 \\
\hline Ajuga bracteosa Wall. & Spina & A & $\mathrm{H}$ & WP & $125 \mathrm{~g}$ pwder is mixed with flour and orally administered for & Abdominal pain & Mandi Bahaudin & Abbas et al.,2013; Hazrat et \\
\hline
\end{tabular}


Adnan et al., Afr J Tradit Complement Altern Med. (2015) 12(5):99-117

http://dx.doi.org/10.4314/ajtcam.v12i5.15

\begin{tabular}{|c|c|c|c|c|c|c|c|c|}
\hline Labiatae & bootei & & & & $2-3$ days. & & & al.,2011 \\
\hline $\begin{array}{l}\text { Luffa cylindrica } \mathrm{L} \text {. } \\
\text { Labiatae }\end{array}$ & Torai & $\mathrm{A}$ & $\mathrm{H}$ & Fr & Fruits are used as vegetable & Stomach pain & Malakand & Alamgeer et al.,2013 \\
\hline $\begin{array}{l}\text { Albizia lebbeck L. } \\
\text { Mimosaceae }\end{array}$ & Sirsirin & A & $\mathrm{T}$ & $\mathrm{B}$ & $\begin{array}{l}\text { Decoction is prepared with T. ammi and C. bengalensis and } \\
\text { given }\end{array}$ & Diarrhea,Dysentery & Southern Punjab & Khan \& Hanif., 2006 \\
\hline $\begin{array}{l}\text { Allium barszczewski } \mathrm{L} \text {. } \\
\text { Alliaceae }\end{array}$ & Kach & B & $\mathrm{S}$ & $\mathrm{L}$ & Eaten raw or cooked as vegetable. & Stomachache & Cholistan & Abbas et al.,2013 \\
\hline $\begin{array}{l}\text { Allium sativum } \mathrm{L} . \\
\text { Alliaceae }\end{array}$ & Lehsan & A & $\mathrm{H}$ & $\mathrm{B}, \mathrm{L}$ & $\begin{array}{l}\text { Bulb and leaves are boiled and the cooled extract } \\
\text { administered against diarrhea, dysentery and vomiting. } \\
\text { Bulbs are fried in mustard oil and can be used for dysentery. }\end{array}$ & $\begin{array}{l}\text { Diarrhoea, Dysentery, } \\
\text { Vomiting }\end{array}$ & $\begin{array}{l}\text { Cholistan, D.I. } \\
\text { Khan }\end{array}$ & $\begin{array}{l}\text { Ballabh \& Chaurasia., 2009; } \\
\text { Rahmatullah et al.,2011; } \\
\text { Mussarat et al.,2014 }\end{array}$ \\
\hline $\begin{array}{l}\text { Aloe vera } \mathrm{L} \text {. } \\
\text { Asphodelaceae }\end{array}$ & $\begin{array}{l}\text { Masloon, } \\
\text { Bistorta }\end{array}$ & A & $\mathrm{H}$ & $\mathrm{P}$ & $\begin{array}{l}\text { Fresh leaf pulp, ghee, sugar and wheat flour cooked } \\
\text { together, make halwa and taken once a day at bedtime for } \\
\text { treatment of constipation. } 1 / 2 \mathrm{~kg} \text { of leaf pulp, salt and T.ammi } \\
\text { are mixed and take orally up to } 1 \text { week for digestive } \\
\text { problems }\end{array}$ & $\begin{array}{l}\text { Constipation } \\
\text { Digestion }\end{array}$ & Bannu & $\begin{array}{l}\text { Awan et al.,2013; Abbasi et } \\
\text { al.,2010 }\end{array}$ \\
\hline $\begin{array}{l}\text { Althea rosea } \mathrm{L} . \\
\text { Malvaceae }\end{array}$ & $\begin{array}{l}\text { Gul e } \\
\text { khaira }\end{array}$ & $\mathrm{P}$ & $\mathrm{H}$ & $\mathrm{R}$ & $\begin{array}{l}\text { Roots are dip in earthen pot for whole night. In morning the } \\
\text { water juice is drunk }\end{array}$ & $\begin{array}{l}\text { Gastro-intestinal } \\
\text { diseases }\end{array}$ & $\begin{array}{l}\text { Leepa Valley, } \\
\text { Muzaffarabad }\end{array}$ & Begum et al., 2014 \\
\hline $\begin{array}{l}\text { Amaranthus hybridus } \mathrm{L} \text {. } \\
\text { Amaranthaceae }\end{array}$ & $\begin{array}{l}\text { Tagalog } \\
\text { Ranjaka }\end{array}$ & A & $\mathrm{H}$ & $\mathrm{S}$ & $\begin{array}{l}\text { Seeds are semi cooked on iron pan, grindered and used as } \\
\text { tea for acidity and stomach disorder }\end{array}$ & $\begin{array}{l}\text { Acidity, Stomach } \\
\text { disorder } \\
\text { Indigestion }\end{array}$ & $\begin{array}{l}\text { Cholistan } \\
\text { Nwazstan }\end{array}$ & $\begin{array}{l}\text { Marwat et al.,2012; Humayun } \\
\text { et al.,2006; Noor et al.,2012 }\end{array}$ \\
\hline $\begin{array}{l}\text { Amaranthus viridis } \\
\text { L.Amaranthaceae }\end{array}$ & $\begin{array}{l}\text { Putkanda, } \\
\text { Ranzaka }\end{array}$ & A & $\mathrm{H}$ & $\mathrm{L}$ & $\begin{array}{l}\text { Leaves are boiled in water with 'gur'and filter through a } \\
\text { cloth, extract use for Expulsion of worms, abdominal pain } \\
\text { and diarrhea. }\end{array}$ & $\begin{array}{l}\text { Worms, Abdominal } \\
\text { pain, Diarrhea }\end{array}$ & $\begin{array}{l}\text { Cholistan } \\
\text { Nwazstan }\end{array}$ & Farooq et al.,2012 \\
\hline $\begin{array}{l}\text { Anthemis cotula } \mathrm{L} \text {. } \\
\text { Asteraceae }\end{array}$ & Sherisht & $\mathrm{P}$ & $\mathrm{H}$ & $\mathrm{F}$ & Flowers are boiled in water and used & $\begin{array}{l}\text { Stomachache, } \\
\text { Gas trouble }\end{array}$ & $\begin{array}{l}\text { Khirthar National } \\
\text { Park }\end{array}$ & Ali \& Qaiser., 2009 \\
\hline $\begin{array}{l}\text { Arisaema flavum Forsk. } \\
\text { Araceae }\end{array}$ & Marjara & $\mathrm{P}$ & $\mathrm{H}$ & $\mathrm{Rh}$ & Rhizome used to kill worm & Worms & $\begin{array}{l}\text { Chapursan Valley } \\
\text { gilgit }\end{array}$ & Hazrat et al.,2011 \\
\hline $\begin{array}{l}\text { Artemasia martima } \mathrm{L} . \\
\text { Asteraceae }\end{array}$ & Gadadhar & A & $\mathrm{S}$ & $F, R$ & $\begin{array}{l}\text { Flower powder and fresh roots Juice is used to expel worms } \\
\text { and stomach pain. }\end{array}$ & Worms & Kohistan & Singh et al.,2012 \\
\hline $\begin{array}{l}\text { Artemisia santolinifolia } \\
\text { Turez. Asteraceae }\end{array}$ & Dron & A & $\mathrm{H}$ & WP & Plant extract is used & Worms & $\begin{array}{l}\text { Khirthar National } \\
\text { Park }\end{array}$ & $\begin{array}{l}\text { Ahmad et al.,2007; Hayat et } \\
\text { al.,2009 }\end{array}$ \\
\hline $\begin{array}{l}\text { Artemisia absinthium } \mathrm{L} \text {. } \\
\text { Asteraceae }\end{array}$ & Sheeh & A & $\mathrm{H}$ & $\mathrm{F}$ & Powder is used & Indigestion & Dir Kohistan Valley & Afzal et al.,2009 \\
\hline $\begin{array}{l}\text { Artemisia parviflora } \\
\text { Roxb. Asteraceae }\end{array}$ & $\begin{array}{l}\text { Kharkhalie } \\
\text { ch }\end{array}$ & A & $\mathrm{S}$ & $\mathrm{S}$ & $\begin{array}{l}\text { One teaspoon powder with one glass water for abdominal } \\
\text { pain. }\end{array}$ & Abdominal pain & $\begin{array}{l}\text { Khirthar National } \\
\text { Park }\end{array}$ & $\begin{array}{l}\text { Ahmad et al.,2007; Khan, } \\
2011\end{array}$ \\
\hline $\begin{array}{l}\text { Artemisia roxburghiana } \\
\text { Wall. Asteraceae }\end{array}$ & $\begin{array}{l}\text { Jangli } \\
\text { ajwain }\end{array}$ & $\mathrm{P}$ & $\mathrm{H}$ & WP & Plant powder is used & Worms & Rawalpindi & $\begin{array}{l}\text { Hayat et al.,2008; Khan et } \\
\text { al.,2012 }\end{array}$ \\
\hline $\begin{array}{l}\text { Asparagus adscendens } \\
\text { Roxb. Asparagaceae }\end{array}$ & $\begin{array}{l}\text { Safid } \\
\text { muesli }\end{array}$ & A & $\mathrm{H}$ & $\mathrm{R}$ & Tuberous roots paste is very effective in dysentery problems & Dysentry & Karak & Mukergee, 2013 \\
\hline $\begin{array}{l}\text { Asparagus racemosus } \\
\text { Wild. Asparagaceae }\end{array}$ & $\begin{array}{l}\text { Shahghand } \\
\text { al/nanoor }\end{array}$ & A & $\mathrm{S}$ & $\mathrm{R}$ & $\begin{array}{l}\text { Corm is used as carminative. Root paste and Extract mixed } \\
\text { with sugar is taken for constipation and dysentery }\end{array}$ & $\begin{array}{l}\text { Carminative, } \\
\text { Constipation } \\
\text { Dysentry }\end{array}$ & $\begin{array}{l}\text { Leepa Valley, } \\
\text { Muzaffarabad }\end{array}$ & $\begin{array}{l}\text { Rahmatullah et al.,2010; Shah } \\
\text { \& Khan., 2006; Shadangi et } \\
\text { al.,2012 }\end{array}$ \\
\hline Astragalus macropterus & & $\mathrm{P}$ & $\mathrm{H}$ & $\mathrm{L}$ & Leaves are use to cure stomachic & Stomachic & Lahore_Islamabad & Wazir et al.,2004 \\
\hline
\end{tabular}


Adnan et al., Afr J Tradit Complement Altern Med. (2015) 12(5):99-117

http://dx.doi.org/10.4314/ajtcam.v12i5.15

\begin{tabular}{|c|c|c|c|c|c|c|c|c|}
\hline DS. Papilionaceae & & & & & & & motorway & \\
\hline $\begin{array}{l}\text { Averrhoa carambola } \mathrm{L} . \\
\text { Oxalidaceae }\end{array}$ & Kamrnga & $\mathrm{A}$ & $\mathrm{S}$ & $\mathrm{F}$ & Fruit is taken orally. & $\begin{array}{l}\text { Dirrhoea } \\
\text { Vomiting }\end{array}$ & Rawalpindi & Rahmattullah et al.,2009 \\
\hline $\begin{array}{l}\text { Azadirachta indica } \mathrm{L} . \\
\text { Meliaceae }\end{array}$ & Neem & $\mathrm{P}$ & $\mathrm{T}$ & $\mathrm{B}$ & $\begin{array}{l}\text { Mixture of bark, lime and salt taken on empty stomach in } \\
\text { the morning for } 7 \text { days }\end{array}$ & Worms & Lakki Marwat & Qureshi et al.,2011 \\
\hline $\begin{array}{l}\text { Barberis lycium Royle. } \\
\text { Berberidaceae }\end{array}$ & $\begin{array}{l}\text { Sumbal, } \\
\text { Toor } \\
\text { Kwaray }\end{array}$ & $\mathrm{P}$ & $\mathrm{S}$ & $\begin{array}{l}\mathrm{R}, \mathrm{L}, \\
\mathrm{F}\end{array}$ & $\begin{array}{l}\text { Two to three teaspoonful of fresh fruit extract is } \\
\text { recommended for stomachache, intestinal problem and } \\
\text { diarrhea. }\end{array}$ & $\begin{array}{l}\text { Stomachache Intestinal } \\
\text { problem Diarrhea }\end{array}$ & $\begin{array}{l}\text { Rawalpindi } \\
\text { Kaghan valley }\end{array}$ & $\begin{array}{l}\text { Ballabh \& Chaurasia., 2009; } \\
\text { Hazrat et al.,2011; Abbasi et } \\
\text { al.,2013 }\end{array}$ \\
\hline $\begin{array}{l}\text { Barleria acanthoides } \\
\text { Vahl. Acanthaceae }\end{array}$ & Thath & $\mathrm{P}$ & $\mathrm{S}$ & $\mathrm{R}$ & Decoction of roots used & Gastrointestinal upset & $\begin{array}{l}\text { Orakzai, Gujar } \\
\text { Khan }\end{array}$ & Ahmad et al.,2007 \\
\hline $\begin{array}{l}\text { Basella alba } \\
\text { Basellaceae }\end{array}$ & Saag & A & $\mathrm{H}$ & $\mathrm{R}, \mathrm{S}$ & Decoction of roots and shoot is given & Intestinal disorder & & Singh et al.,2012 \\
\hline $\begin{array}{l}\text { Bergenia ciliata Sternb. } \\
\text { Saxifragaceae }\end{array}$ & $\begin{array}{l}\text { Batpia,Gat } \\
\text { panra } \\
\text { Zakhm-e- } \\
\text { Hayat }\end{array}$ & $\mathrm{P}, \mathrm{A}$ & $\mathrm{S}$ & $\mathrm{R}, \mathrm{Rh}$ & $\begin{array}{l}\text { The root is used in the treatment of diarrhea and digestive } \\
\text { disorder. Rhizome is crushed and used in all kinds of } \\
\text { stomach and duodenal ulcer. }\end{array}$ & $\begin{array}{l}\text { Diarrhea,Indigestion,St } \\
\text { omachUlcer, Internal } \\
\text { infections }\end{array}$ & $\begin{array}{l}\text { Jalalpur Jattan, } \\
\text { Gujrat }\end{array}$ & $\begin{array}{l}\text { Arshad et al.,2014; Begum et } \\
\text { al.,2014; Humayun et } \\
\text { al.,2006; Chaudary et al.,2013 }\end{array}$ \\
\hline $\begin{array}{l}\text { Bergenia ligulata Wall. } \\
\text { Saxifragaceae }\end{array}$ & Gatpanra & $\mathrm{P}$ & $\mathrm{H}$ & $\mathrm{Rh}, \mathrm{R}$ & $\begin{array}{l}10 \mathrm{~g} \text { of rhizome juice is taken orally with molasses, twice a } \\
\text { day for 3-4 days as anthelmentic. Roots infusion for } \\
\text { dysentery and stomach disorders. }\end{array}$ & $\begin{array}{l}\text { Worms, Dysentry } \\
\text { Stomach disorder }\end{array}$ & $\begin{array}{l}\text { Jalalpur Jattan, } \\
\text { Gujrat }\end{array}$ & Gurav \& Gurav., 2014 \\
\hline $\begin{array}{l}\text { Boerhaavia diffusa } \mathrm{L} \text {. } \\
\text { Nyctaginaceae }\end{array}$ & Punara & $\mathrm{P}$ & $\mathrm{H}$ & WP & Extract with raw sugar is use $\mathrm{d}$ & Stomach disorder & Karak & $\begin{array}{l}\text { Khan \& Hanif., 2006; Kumar } \\
\text { \& Bhagut., } 2012\end{array}$ \\
\hline $\begin{array}{l}\text { Boerhavia procumbens } \\
\text { Roxb. Nyctaginaceae }\end{array}$ & Punarnava & $\mathrm{P}$ & $\mathrm{H}$ & $\mathrm{R}$ & Decoction is used. & $\begin{array}{l}\text { Dyspepsia, Abdominal } \\
\text { pain }\end{array}$ & $\begin{array}{l}\text { Takht-e-Nasrati, } \\
\text { Karak }\end{array}$ & $\begin{array}{l}\text { Marwat et al.,2012; Qureshi et } \\
\text { al.,2011 }\end{array}$ \\
\hline $\begin{array}{l}\text { Brassica campestris } \mathrm{L} . \\
\text { Brassicaceae }\end{array}$ & $\begin{array}{l}\text { Tepar, } \\
\text { Sarson }\end{array}$ & $\mathrm{A}$ & $\mathrm{H}$ & $\mathrm{S}$ & $\begin{array}{l}100 \mathrm{~g} \text { seeds are ground, and then powder is mixed with eggs } \\
\text { and orally administered for } 2-3 \text { days }\end{array}$ & Stomach infection & Dir, kohistan valley & $\begin{array}{l}\text { Abbas et al.,2013; Hassan et } \\
\text { al.,2014 }\end{array}$ \\
\hline $\begin{array}{l}\text { Bunium persicum Boiss. } \\
\text { Umbelliferae }\end{array}$ & Kala zera & $\mathrm{A}$ & $\mathrm{H}$ & Fr, S & Fresh fruits and dried seeds are used & $\begin{array}{l}\text { Gastrointestinal } \\
\text { disturbance } \\
\text { Dysentry }\end{array}$ & Chitral & Sharma et al.,2011 \\
\hline $\begin{array}{l}\text { Callicarpa Macrophylla } \\
\text { Vahl. Verbenaceae }\end{array}$ & Daya & $\mathrm{A} / \mathrm{P}$ & $\mathrm{S}$ & $\mathrm{R}$ & Root juice is taken orally & Indigestion & $\begin{array}{l}\text { Valley Alladand } \\
\text { Dehri, Malakand }\end{array}$ & Jones \& Kinghorne., 2008 \\
\hline $\begin{array}{l}\text { Calotropis procera } \\
\text { Willd. Asclepiadaceae }\end{array}$ & $\begin{array}{l}\text { Spulmaey, } \\
\text { Ak }\end{array}$ & A & $\mathrm{S}$ & F, L & $\begin{array}{l}\text { 50gm flower powder along with } P \text {. somniferum, Eletteria } \\
\text { cardomomum }(20 \mathrm{~g} \text { each) used. Warmed leaves are tied on } \\
\text { abdomen for colic }\end{array}$ & $\begin{array}{l}\text { Cholera, Dysentery, } \\
\text { Colic }\end{array}$ & $\begin{array}{l}\text { Kaghan } \\
\text { valleyBugrote }\end{array}$ & $\begin{array}{l}\text { Khan et al.,2012; Punjani, } \\
2002\end{array}$ \\
\hline $\begin{array}{l}\text { Cannabis sativa } \mathrm{L} \text {. } \\
\text { Cannabaceae }\end{array}$ & Bhang. & A & $\mathrm{H}$ & WP & $\begin{array}{l}1 \mathrm{~kg} \text { of dried leaves, floral buds and seeds are ground and } \\
\text { mixed with wheat flour, salt and water use for } 10-15 \text { days }\end{array}$ & $\begin{array}{l}\text { Abdominal swelling } \\
\text { Indigestion }\end{array}$ & Upper Dir & $\begin{array}{l}\text { Manan et al.,2007; Abbasi et } \\
\text { al.,2013; Iqbal et al.,2011 }\end{array}$ \\
\hline $\begin{array}{l}\text { Capparis decidua } \\
\text { (Forsk) Edgew. } \\
\text { Capparidaceae }\end{array}$ & Karir & $\mathrm{P}$ & $\mathrm{S}$ & $\begin{array}{l}\mathrm{AP} \\
\mathrm{F}, \mathrm{B}\end{array}$ & $\begin{array}{l}\text { Decoction of } 1 / 2 \mathrm{~kg} \text { of fresh aerial parts and fruits is used for } \\
5-6 \text { days }\end{array}$ & $\begin{array}{l}\text { Indigestion } \\
\text { Carminative } \\
\text { Stomachache }\end{array}$ & Upper Dir & $\begin{array}{l}\text { Abbasi et al.,2013; Marwat et } \\
\text { al.,2011 }\end{array}$ \\
\hline $\begin{array}{l}\text { Capsicum annuиm } \mathrm{L} \text {. } \\
\text { Solanaceae }\end{array}$ & Merchiki & A & $\mathrm{H}$ & $\mathrm{F}$ & Fresh fruit with table salt and gur is used & Stomach disorder & $\begin{array}{l}\text { Poonch Valley Azad } \\
\text { Kashmir }\end{array}$ & Khan \& Hanif., 2006 \\
\hline $\begin{array}{l}\text { Caralluma edulis } \\
\text { Benth. Asclepiadaceae }\end{array}$ & Pamankai & A & $\mathrm{H}$ & WP & Fresh plant is used & Parasitic diseases & $\begin{array}{l}\text { Bugrote valleys } \\
\text { Gilgit }\end{array}$ & Safa et al.,2012 \\
\hline Caralluma tuberculata & Marmoot, & $\mathrm{A}$ & $\mathrm{H}$ & WP & Plant powder is used & Dysentery, Stomach & Kaghan valley & Tareen et al.,2010; Ullah et \\
\hline
\end{tabular}


Adnan et al., Afr J Tradit Complement Altern Med. (2015) 12(5):99-117

http://dx.doi.org/10.4314/ajtcam.v12i5.15

\begin{tabular}{|c|c|c|c|c|c|c|c|c|}
\hline Brown. Asclepiadaceae & Pawany & & & & & pain, Constipation & & al., 2010 \\
\hline $\begin{array}{l}\text { Carissa opaca Stapf. } \\
\text { Apocynaceae }\end{array}$ & Granda & $\mathrm{A}$ & $\mathrm{S}$ & $\mathrm{R}$ & $\begin{array}{l}\text { Root powder mixed with pericarp of Mangifera indica and } \\
\text { used as wormicide }\end{array}$ & Worms & Karak & $\begin{array}{l}\text { Khan \& Hanif., 2006; } \\
\text { Mahmood et al.,2011 }\end{array}$ \\
\hline $\begin{array}{l}\text { Carthamus oxyacantha } \\
\text { M.Bieb. Asteraceae }\end{array}$ & kareeza & $\mathrm{A}$ & $\mathrm{H}$ & $\begin{array}{l}\mathrm{L} \\
\mathrm{S}\end{array}$ & $\begin{array}{l}\text { Decoction of leaves is used against dysentery. Seeds are } \\
\text { used for stomach pain. }\end{array}$ & $\begin{array}{l}\text { Dysentry } \\
\text { Stomachache }\end{array}$ & $\begin{array}{l}\text { lahore-islamabad } \\
\text { motoway,Khirthar } \\
\text { National Park }\end{array}$ & Alamgeer et al.,2013 \\
\hline $\begin{array}{l}\text { Carum carvi } \mathrm{L} \text {. } \\
\text { Umbelliferae }\end{array}$ & Hojoj & $P$ & $\mathrm{H}$ & $\mathrm{F}, \mathrm{S}$ & $\begin{array}{l}\text { Fruit decoction use to cure colic. Few seeds are taken } \\
\text { directly with fresh water before sleeping as carminative and } \\
\text { promote digestion. }\end{array}$ & $\begin{array}{l}\text { Colic } \\
\text { Carminative Indigestion }\end{array}$ & Kala Bagh & $\begin{array}{l}\text { Ballabh \& Chaurasia., 2009; } \\
\text { Ahmad et al.,2007; Zid et al. } \\
\text { AbouZid \& Mohamed,2011 }\end{array}$ \\
\hline $\begin{array}{l}\text { Chenopodium } \\
\text { ambrosioides } \mathrm{L} \text {. } \\
\text { Chenopodiaceae }\end{array}$ & Ranzekka & A & $\mathrm{H}$ & $\mathrm{F}$ & $\begin{array}{l}\text { The dried ripe fruits are crushed into powder, which is taken } \\
\text { orally with water }\end{array}$ & Dyspepsia & Khushab & $\begin{array}{l}\text { Khan et al.,2013; Adnan et } \\
\text { al.,2014 }\end{array}$ \\
\hline $\begin{array}{l}\text { Chenopodium botrys } \mathrm{L} \text {. } \\
\text { Chenopodiaceae }\end{array}$ & $\begin{array}{l}\text { Skha } \\
\text { kharawa }\end{array}$ & A & $\mathrm{H}$ & WP & Aqueous extract of the plant is used & Stomachache & Khushab & Barkattullah et al.,2009 \\
\hline $\begin{array}{l}\text { Chenopodium morale } \mathrm{L} \text {. } \\
\text { Chenopodiaceae }\end{array}$ & Wereaij & $\mathrm{A}$ & $\mathrm{S}$ & WP & Decoction is used & Helminth and Laxation & Upper Dir & $\begin{array}{l}\text { Khan et al.,2013; Hamayun, } \\
2007\end{array}$ \\
\hline $\begin{array}{l}\text { Chrozophora tinctoria } \\
\text { L. Euphorbiaceae }\end{array}$ & Neeli Booti & A & $\mathrm{S}$ & $\mathrm{L}$ & Leaves are boiled in water and given orally & Stomachic & $\begin{array}{l}\text { Makerwal \& Gulla } \\
\text { Khel }\end{array}$ & Qureshi et al.,2011 \\
\hline $\begin{array}{l}\text { Cichorium intybus } \mathrm{L} . \\
\text { Asteraceae }\end{array}$ & $\begin{array}{l}\text { Hund, } \\
\text { Kasni, } \\
\text { Gojri }\end{array}$ & $\mathrm{A}$ & $\mathrm{H}$ & $\begin{array}{l}\mathrm{F}, \mathrm{L}, \\
\mathrm{R}\end{array}$ & Decoction of plant is taken & $\begin{array}{l}\text { Gas trouble Diarrhoea } \\
\text { Constipation } \\
\text { Stomachache }\end{array}$ & $\begin{array}{l}\text { Kohistan Khirthar } \\
\text { National Park }\end{array}$ & $\begin{array}{l}\text { Abbas et al.,2013; Awan et } \\
\text { al.,2013; Fakir et al.,2009 }\end{array}$ \\
\hline $\begin{array}{l}\text { Cistanche tubulosa } \\
\text { (Schenk) Wight. } \\
\text { Orobanchaceae }\end{array}$ & Kasi & $\mathrm{A}$ & $\mathrm{H}$ & WP & $\begin{array}{l}\text { Powder of the plant is given in diarrhea. Whole plant } \\
\text { decoction used to cure the gastric ulcer, vomiting and } \\
\text { diarrhea. }\end{array}$ & $\begin{array}{l}\text { Diarrhea } \\
\text { Ulcer, Vomiting, } \\
\text { Diarrhea }\end{array}$ & $\begin{array}{l}\text { Malakand Valley } \\
\text { Dir (Lower) }\end{array}$ & Qureshi et al.,2010 \\
\hline $\begin{array}{l}\text { Citrullus colocynthis } \mathrm{L} . \\
\text { Cucurbitaceae }\end{array}$ & $\begin{array}{l}\text { Maraghona } \\
\text { e }\end{array}$ & $\mathrm{P}$ & $\mathrm{H}$ & F, S & $\begin{array}{l}\text { Fruit and root pills use for constipation and piles. Juice of } \\
\text { plant and } C \text {. procera is used up to a week. } 100 \mathrm{~g} \text { fruit is } \\
\text { crushed, mixed in Aloe vera pulp and taken for constipation } \\
\text { for } 2-3 \text { days. }\end{array}$ & $\begin{array}{l}\text { Stomachache } \\
\text { Constipation, Piles } \\
\text { Indigestion, Gas trouble } \\
\text { and worms }\end{array}$ & $\begin{array}{l}\text { Attock, Kaghan } \\
\text { Valley }\end{array}$ & $\begin{array}{l}\text { Hayat et al.,2008; Abbasi et } \\
\text { al.,2013 }\end{array}$ \\
\hline $\begin{array}{l}\text { Convolvulus arvensis } \mathrm{L} \text {. } \\
\text { Convolvulaceae }\end{array}$ & $\begin{array}{l}\text { Leli / weli } \\
\text { Pirwathia }\end{array}$ & $\mathrm{P}$ & $\mathrm{H}$ & WP & $\begin{array}{l}\text { Dried roots are grinded and take } 1-2 \text { spoons as } \\
\text { Purgative. } 1 \mathrm{~kg} \text { fresh plant is crushed along with sugar and } \\
\text { water and given orally for } 3-4 \text { days }\end{array}$ & $\begin{array}{l}\text { Purgation } \\
\text { Constipation }\end{array}$ & $\begin{array}{l}\text { Dir Kohistan } \\
\text { Valley, Punjab }\end{array}$ & $\begin{array}{l}\text { Manan et al.,2007; Arshad et } \\
\text { al.,2014; Hussain et al.,2010; } \\
\text { Adnan et al.,2014 }\end{array}$ \\
\hline $\begin{array}{l}\text { Cordia myxa } \mathrm{L} . \\
\text { Boraginaceae }\end{array}$ & Lasora & A & $\mathrm{T}$ & $\begin{array}{l}\text { B, L, } \\
\text { F }\end{array}$ & Plant parts are used & Stomachache & Swat & Marwat et al.,2012 \\
\hline $\begin{array}{l}\text { Cordia obliqua Willd. } \\
\text { Boraginaceae }\end{array}$ & Lasoora, & A & $\mathrm{T}$ & $\begin{array}{l}\text { S, St, } \\
\text { B }\end{array}$ & $\begin{array}{l}\text { Decoction of seeds stem and bark is administered orally for } \\
10-15 \text { days }\end{array}$ & Ulcer & Rawalpindi & $\begin{array}{l}\text { Abbas et al.,2013; Krishna et } \\
\text { al.,2014 }\end{array}$ \\
\hline $\begin{array}{l}\text { Coriandrum sativum } \mathrm{L} . \\
\text { Umbelliferae }\end{array}$ & Dhania & $\mathrm{P}$ & $\mathrm{H}$ & $\mathrm{L}$ & Roast their leaves and take with water orally & Carminative & $\begin{array}{l}\text { Valley Alladand } \\
\text { Malakand }\end{array}$ & Adnan et al.,2014 \\
\hline $\begin{array}{l}\text { Croton } \\
\text { bonplandianusBail. } \\
\text { Euphorbiaceae }\end{array}$ & Mirch boti & $\mathrm{P}$ & $\mathrm{H}$ & $\mathrm{R}$ & Crushed roots are taken & Gastric diseases & $\begin{array}{l}\text { Lahore_Islamabad } \\
\text { motorway }\end{array}$ & $\begin{array}{l}\text { Ullah et al.,2010; Mahmood et } \\
\text { al.,2011 }\end{array}$ \\
\hline Cucumis melo L. & Chibbar & A & $\mathrm{H}$ & $\mathrm{F}, \mathrm{L}$ & The ripened fruit is cut into pieces and dried, which is used & Laxative, Indigestion & Attock, Kaghan & Hussain et al.,2010; Abbasi et \\
\hline
\end{tabular}


Adnan et al., Afr J Tradit Complement Altern Med. (2015) 12(5):99-117

http://dx.doi.org/10.4314/ajtcam.v12i5.15

\begin{tabular}{|c|c|c|c|c|c|c|c|c|}
\hline Cucurbitaceae & & & & & as a condiment in cooking vegetables & & $\begin{array}{l}\text { Valley, } \\
\text { Malakand }\end{array}$ & al.,2013; Arshad et al.,2011 \\
\hline $\begin{array}{l}\text { Cucurbita pepo L. } \\
\text { Cucurbitaceae }\end{array}$ & $\begin{array}{l}\text { Gharangy } \\
\text { Kadoo }\end{array}$ & A & $\mathrm{H}$ & F & $\begin{array}{l}\text { Fruit pieces are boiled in } 1.5 \text { liters of water. Add salt and } \\
\text { black pepper for taste and decoction used. Seeds are eaten } \\
\text { raw as anti-helminthic }\end{array}$ & $\begin{array}{l}\text { Intestinal, Gastric } \\
\text { disorder } \\
\text { Helminths }\end{array}$ & Attock, Malakand & $\begin{array}{l}\text { Hayat et al.,2008; Mustafa et } \\
\text { al.,2012 }\end{array}$ \\
\hline $\begin{array}{l}\text { Cyperus nevieus Retz. } \\
\text { Cyperaceae }\end{array}$ & Deela & A & $\mathrm{H}$ & $\mathrm{R}$ & Crushed root is taken & Stomachic & Malakand & Ahmad, 2007 \\
\hline $\begin{array}{l}\text { Cyperus rotundus } \\
\text { L.Cyperaceae }\end{array}$ & $\begin{array}{l}\text { Nagermoth } \\
\text { a, } \\
\text { Dilla, } \\
\text { Kabah }\end{array}$ & $\mathrm{P}$ & $\mathrm{H}$ & $\mathrm{R}, \mathrm{Rh}$ & $\begin{array}{l}\text { Decoction of root and equal quantity of } M \text {. piperata given } \\
\text { for } 4-5 \text { days. } 5 \mathrm{~g} \text { of rhizome powder is taken with milk daily } \\
3 \text { times a day for } 2-3 \text { days to cure dysentery. }\end{array}$ & $\begin{array}{l}\text { Cholera, Dysentry } \\
\text { Indigestion }\end{array}$ & $\begin{array}{l}\text { Malakand, } \\
\text { Punjab,Bannu, } \\
\text { SouthWaziristan } \\
\text { Agency }\end{array}$ & $\begin{array}{l}\text { Hussain et al.,2010; Qureshi } \\
\text { et al.,2010; Gulshan et } \\
\text { al.,2012; Biswas et al.,2011 }\end{array}$ \\
\hline $\begin{array}{l}\text { Cyperus scariosus R.Br. } \\
\text { Cyperaceae }\end{array}$ & Tarkh & $P$ & $\mathrm{H}$ & WP & Powder of plant or fresh plant is used & Worms & $\begin{array}{l}\text { Kadhi Areas of } \\
\text { khushab }\end{array}$ & Afzal et al.,2009 \\
\hline $\begin{array}{l}\text { Dalbergia sissoo Roxb. } \\
\text { Papilionaceae }\end{array}$ & $\begin{array}{l}\text { Shisham, } \\
\text { Shawa }\end{array}$ & $\mathrm{P}$ & $\mathrm{T}$ & $\mathrm{B}, \mathrm{L}$ & $\begin{array}{l}\text { Crushed bark as remedy for the abdominal pain. } \\
\text { Deecocotion of } 1 / 2 \mathrm{~kg} \text { fresh leaves, } 200 \mathrm{~g} \text { linseeds taken } \\
\text { orally for } 8-10 \text { days for constipation. }\end{array}$ & $\begin{array}{l}\text { Abdominal pain } \\
\text { Constipation }\end{array}$ & $\begin{array}{l}\text { Northern areas } \\
\text { Miandam Valley, } \\
\text { Swat, }\end{array}$ & $\begin{array}{l}\text { Abbas et al.,2013; Hassan et } \\
\text { al.,2014 }\end{array}$ \\
\hline $\begin{array}{l}\text { Datura stramonium } \mathrm{L} . \\
\text { Solanaceae }\end{array}$ & Batura & A & $\mathrm{H}$ & $\mathrm{L}$ & Leaf juice is used & Stomachache & Karak & Manan et al.,2007 \\
\hline $\begin{array}{l}\text { Debregeasia longifolia } \\
\text { Rendle. Urticaceae }\end{array}$ & Sandari. & $\mathrm{P}$ & $\mathrm{H}$ & $\mathrm{L}$ & Tender leaves are taken as vegetable & Dysentry & $\begin{array}{l}\text { Valley Alladand } \\
\text { Dehri,Malakand }\end{array}$ & $\begin{array}{l}\text { Khan et al.,2012; Majumdar } \\
\text { \& Datta., } 2013\end{array}$ \\
\hline $\begin{array}{l}\text { Diospyros lotus L. } \\
\text { Ebenaceae }\end{array}$ & Amlok & A & $\mathrm{T}$ & $\mathrm{F}$ & Decoction of ripened fruit is used & Dysentry & Tehsil Birmal, SWA & Sher \& Hussain., 2009 \\
\hline $\begin{array}{l}\text { Dodonaea viscosa Jacq. } \\
\text { Sapindaceae }\end{array}$ & Sanatha & $\mathrm{B} / \mathrm{P} / \mathrm{A}$ & $\mathrm{S}$ & $\mathrm{L}$ & Grind dried leaves into powder and take orally with water & Stomach acidity & $\begin{array}{l}\text { Jalalpur Jattan, } \\
\text { Gujrat }\end{array}$ & $\begin{array}{l}\text { Hayat et al.,2008; Hussain et } \\
\text { al.,2010 }\end{array}$ \\
\hline $\begin{array}{l}\text { Echinops echinatus } \\
\text { Roxb. Asteraceae }\end{array}$ & Tik. & $\mathrm{B}$ & $\mathrm{H}$ & WP & Whole plant infusion use orally & Dyspepsia & Rawalpindi & Murad et al.,2012 \\
\hline $\begin{array}{l}\text { Eclipta alba } \mathrm{L} . \\
\text { Asteraceae }\end{array}$ & Tik. & A & $\mathrm{T}$ & $\mathrm{R}$ & Root powder or decoction is taken & Nausea and Vomiting & Rawalpindi & Wariss et al.,2014 \\
\hline $\begin{array}{l}\text { Elaeagnus angustifolia } \\
\text { L. Elaeagnaceae }\end{array}$ & Sersang & $P$ & $\mathrm{~T}$ & $\begin{array}{l}\mathrm{F}, \mathrm{L}, \\
\mathrm{Fl}\end{array}$ & Plant parts are used in powder form & $\begin{array}{l}\text { Ulcer, Diarrhea and } \\
\text { Dyspepsia }\end{array}$ & Lakimarwat & $\begin{array}{l}\text { Khan, 2011; Mardaninejad et } \\
\text { al.,2013 }\end{array}$ \\
\hline $\begin{array}{l}\text { Ephedra } \\
\text { gerardianaWall ex } \\
\text { Stapf. Ephedraceae }\end{array}$ & Soane & A & $\mathrm{S}$ & $\mathrm{L}$ & The leaves and stem extract is used & Stomachache & LakkiMarwat & Hadi et al.,2013 \\
\hline $\begin{array}{l}\text { Eruca sativaMiller. } \\
\text { Brassicaceae }\end{array}$ & Tarameera & $\mathrm{A}$ & $\mathrm{H}$ & $\mathrm{S}$ & $\begin{array}{l}200 \mathrm{ml} \text { seed oil is mixed with } 200 \mathrm{~g} \text { of sugar orally used for } \\
4-5 \text { days }\end{array}$ & Dysentry & $\begin{array}{l}\text { Attock, Dir, } \\
\text { kohistan valley }\end{array}$ & $\begin{array}{l}\text { Murad et al.,2013; Abbas et } \\
\text { al.,2013 }\end{array}$ \\
\hline $\begin{array}{l}\text { Euphorbia hirta L. } \\
\text { Euphorbiaceae }\end{array}$ & Titra & $P$ & $\mathrm{H}$ & WP & $\begin{array}{l}\text { Extract of milky juice is used orally for infants } \\
\text { as carminative }\end{array}$ & Carminative & $\begin{array}{l}\text { Lakki Marwat, } \\
\text { Makerwal \& Gulla } \\
\text { Khel }\end{array}$ & Adnan et al.,2014 \\
\hline $\begin{array}{l}\text { Fagonia bruguieri DC. } \\
\text { Zygophyllaceae }\end{array}$ & $\begin{array}{l}\text { Drummahu } \\
\text {. }\end{array}$ & $\mathrm{P}$ & $\mathrm{S}$ & $\begin{array}{l}\text { WP } \\
\text { L, Fr }\end{array}$ & $\begin{array}{l}\text { The plant powder is used for gastritis, dyspepsia, } \\
\text { flatulenceand vomiting. Leaves and fruit's decoction is used }\end{array}$ & $\begin{array}{l}\text { Gastritis, Dyspepsia, } \\
\text { Vomiting } \\
\text { Abdominal pain }\end{array}$ & $\begin{array}{l}\text { Malam Jabba } \\
\text { valley, Swat }\end{array}$ & $\begin{array}{l}\text { Wariss et al.,2014; Noor et } \\
\text { al.,2012 }\end{array}$ \\
\hline $\begin{array}{l}\text { Fagonia cretica } \mathrm{L} . \\
\text { Zygophyllaceae }\end{array}$ & $\begin{array}{l}\text { Spelaghzii } \\
\text { Shin }\end{array}$ & A & $\mathrm{H}$ & WP & The extract of whole plant is used & Inflammation & Galyat & Murad et al.,2013 \\
\hline
\end{tabular}


Adnan et al., Afr J Tradit Complement Altern Med. (2015) 12(5):99-117

http://dx.doi.org/10.4314/ajtcam.v12i5.15

\begin{tabular}{|c|c|c|c|c|c|c|c|c|}
\hline $\begin{array}{l}\text { Fagonia indica } \text { Burm. } \\
\text { Zygophyllaceae }\end{array}$ & Dhaman & $\mathrm{A}$ & $\mathrm{H}$ & WP & Grind the whole plant and take $2-3$ spoons as purgative. & Purgative & Gallyat & $\begin{array}{l}\text { Abbas et al.,2013; Adnan et } \\
\text { al.,2014 }\end{array}$ \\
\hline $\begin{array}{l}\text { Ferula assafoetida } \mathrm{L} . \\
\text { Umbelliferae }\end{array}$ & Sup & $\mathrm{P}$ & $\mathrm{H}$ & $\mathrm{R}, \mathrm{La}$ & Latex, root powder use for parasite repellent. & Parasite & Chitral & Safa et al.,2012 \\
\hline $\begin{array}{l}\text { Ficus carica } \\
\text { L.Moraceae }\end{array}$ & Inzar & $\mathrm{A}$ & $\mathrm{T}$ & $\mathrm{L}, \mathrm{F}$ & $\begin{array}{l}\text { Fruit is eaten daily twice a day to cure constipation. Leaves } \\
\text { and fruit decoction use orally for dysentery. }\end{array}$ & $\begin{array}{l}\text { Constipation } \\
\text { Dysentry }\end{array}$ & $\begin{array}{l}\text { Tunglai Mountain } \\
\text { Baffa Mansehra }\end{array}$ & Barkatullah et al.,2009 \\
\hline $\begin{array}{l}\text { Foeniculum vulgare } \\
\text { Mill. Umbelliferae }\end{array}$ & Sonf & $\mathrm{P}$ & $\mathrm{H}$ & $\mathrm{F}$ & $\begin{array}{l}250 \mathrm{~g} \text { fruits powder mix with equal quantity of pomegranate } \\
\text { powder and one teaspoon thrice in a day before and after } \\
\text { meal.decoction of fennel fruit, fresh mint leaves and green } \\
\text { used for vomiting }\end{array}$ & $\begin{array}{l}\text { Indigestion, Chronic } \\
\text { dyspepsia, Gastritis } \\
\text { Dysentry, Diarrhea } \\
\text { Vomiting }\end{array}$ & $\begin{array}{l}\text { Chitral } \\
\text { Kala Bagh }\end{array}$ & $\begin{array}{l}\text { Mussarat et al.,2014; Afzal et } \\
\text { al.,2009; Kumar et al.,2009 }\end{array}$ \\
\hline $\begin{array}{l}\text { Fumaria indica } \\
\text { Hausskn. Fumariaceae }\end{array}$ & $\begin{array}{l}\text { Shahtora } \\
\text { Pita papara, }\end{array}$ & $\mathrm{A}$ & $\mathrm{T}$ & WP & $\begin{array}{l}\text { The juices mixed with honey useful in constipation. Whole } \\
\text { plant is boiled in water and decoction is used for } \\
\text { constipation. }\end{array}$ & Constipation & $\begin{array}{l}\text { Sothern Punjab, } \\
\text { Northern Areas }\end{array}$ & $\begin{array}{l}\text { Manan et al.,2007; Noor et } \\
\text { al.,2012; Hussain et al.,2008; } \\
\text { Jan et al.,2008 }\end{array}$ \\
\hline $\begin{array}{l}\text { Gardenia jasminoides } \\
\text { Ellis. Rubiaceae }\end{array}$ & Chandna & $\mathrm{P}$ & $\mathrm{S}$ & $\mathrm{L}, \mathrm{F}$ & Leaves and flower are use in stomach ache. & Stomachache & Balochistan & Hussain et al.,2010 \\
\hline $\begin{array}{l}\text { Gentiana decumbens } \\
\text { Wall. Gentianaceae }\end{array}$ & Neeli Booti & A & $\mathrm{T}$ & WP & A tincture of this plant has been prepared and used & Stomachache & $\begin{array}{l}\text { District Sialkot, } \\
\text { Punjab }\end{array}$ & Qureshi et al.,2007 \\
\hline $\begin{array}{l}\text { Gentiana kurro Royle. } \\
\text { Gentianaceae }\end{array}$ & Trayamana & $\mathrm{P}$ & $\mathrm{H}$ & $\mathrm{R}$ & Roots are dried to make powder and taken along desi ghee & Stomachache & Pakistan & Begum et al.,2014 \\
\hline $\begin{array}{l}\text { Gentianodes olivier } \\
\text { Griseb. Gentianaceae }\end{array}$ & Nilkant & $\mathrm{P}$ & $\mathrm{H}$ & $\mathrm{R}$ & Decoction of root is used & Stomachache & Pakistan & Tareen et al.,2010 \\
\hline $\begin{array}{l}\text { Gisekia pharnaceoides } \\
\text { L. Aizoaceae }\end{array}$ & Aluuka & B & $\mathrm{H}$ & WP & Fresh parts are used & $\begin{array}{l}\text { Indigestion and } \\
\text { parasites }\end{array}$ & Sialkot & Safa et al.,2012 \\
\hline $\begin{array}{l}\text { Grewia tenax (Forssk.) } \\
\text { Tiliaceae }\end{array}$ & Anzirai & A & $\mathrm{S}$ & $\mathrm{R}, \mathrm{B}$ & Decoction of root and bark powder is used & Dysentry & $\begin{array}{l}\text { Booni Valley, } \\
\text { Chitral }\end{array}$ & Patel et al.,2013 \\
\hline $\begin{array}{l}\text { Haloxylon griffithii } \\
\text { Boiss. Chenopodiaceae }\end{array}$ & Bundi & A & $\mathrm{S}$ & WP & The whole plant is boiled in water and the decoction is used & Stomachache & Dir & Tareen et al.,2010 \\
\hline $\begin{array}{l}\text { Haloxylon recurvum } \\
\text { Bioss. Chenopodiaceae }\end{array}$ & Khar & A & $\mathrm{S}$ & WP & Ash powder is used & $\begin{array}{l}\text { Flatulence, Dyspepsia, } \\
\text { Constipation } \\
\text { Hemorrhoids }\end{array}$ & Upper Dir & Waris et al.,2014 \\
\hline $\begin{array}{l}\text { Heliotropium strigosum } \\
\text { L. Boraginaceae }\end{array}$ & $\begin{array}{l}\text { Kharsan } \\
\text { Gorakh pan }\end{array}$ & A & $\mathrm{H}$ & WP & $\begin{array}{l}\text { Infusion used for cooling purposes. Add sugar to infusion } \\
\text { for taste. }\end{array}$ & Inflammation & $\begin{array}{l}\text { Swat,North } \\
\text { Waziristan }\end{array}$ & $\begin{array}{l}\text { Hayat et al.,2008; Iqbal et } \\
\text { al.,2011 }\end{array}$ \\
\hline $\begin{array}{l}\text { Hertia intermedia } \\
\text { Boiss. Asteraceae }\end{array}$ & Manguli & A & $\mathrm{H}$ & $\mathrm{L}$ & Leaves are boiled in water and the decoction is used & Stomachache & Nara desert & Tareen et al.,2010 \\
\hline $\begin{array}{l}\text { Hippophae rhamnoides } \\
\text { L. Elaeagnaceae }\end{array}$ & Bera, Sarla & A & $\mathrm{T}$ & $\mathrm{F}$ & Syrup is prepared from sour fruit and used & Diarrhea & Tehsil Birmal, SWA & Afzal et al.,2009 \\
\hline $\begin{array}{l}\text { Indigofera gerardiana } \\
\text { Wall.exBaker. } \\
\text { Papilionaceae }\end{array}$ & $\begin{array}{l}\text { Ghore jey } \\
\text { Nili veda }\end{array}$ & $\mathrm{P}$ & $\mathrm{T}$ & $\mathrm{L}$ & Leaves juice is used & Diarrhea, Dysentry & $\begin{array}{l}\text { Lahore_Islamabad } \\
\text { motorway, Southern } \\
\text { Punjab }\end{array}$ & Kumari et al.,2013 \\
\hline $\begin{array}{l}\text { Inula grandiflora Willd. } \\
\text { Asteraceae }\end{array}$ & Poshkara & A & $\mathrm{H}$ & $\mathrm{Rh}$ & Both powdered and fresh rhizome is used & Gastric disorder & $\begin{array}{l}\text { Khirthar National } \\
\text { Park }\end{array}$ & Khan et al.,2013 \\
\hline
\end{tabular}


Adnan et al., Afr J Tradit Complement Altern Med. (2015) 12(5):99-117

http://dx.doi.org/10.4314/ajtcam.v12i5.15

\begin{tabular}{|c|c|c|c|c|c|c|c|c|}
\hline $\begin{array}{l}\text { Inula racemosa H.K. } \\
\text { Asteraceae }\end{array}$ & poshkar & $\mathrm{P}$ & $\mathrm{H}$ & $\mathrm{R}$ & Roots are use to cure stomachic and carminative. & Carmination & $\begin{array}{l}\text { Khirthar National } \\
\text { Park }\end{array}$ & Wazir et al., 2004 \\
\hline $\begin{array}{l}\text { Jatropha hastate Jacq. } \\
\text { Euphorbiaceae }\end{array}$ & Arind & $\mathrm{P}$ & $\mathrm{T}$ & $\mathrm{L}, \mathrm{S}$ & $\begin{array}{l}\text { Decoctions of the leaves used for stomachache. Seeds oil is } \\
\text { used as purgative. }\end{array}$ & $\begin{array}{l}\text { Stomachache } \\
\text { Purgative }\end{array}$ & $\begin{array}{l}\text { Makerwal \& Gulla } \\
\text { Khel, swat }\end{array}$ & Ahmad, 2007 \\
\hline $\begin{array}{l}\text { Justicia adhatoda } \mathrm{L} . \\
\text { Acanthaceae }\end{array}$ & $\begin{array}{l}\text { Bhekkar, } \\
\text { Vasaka, } \\
\text { Baza }\end{array}$ & $\mathrm{B}$ & $\mathrm{S}$ & $\mathrm{L}$ & $\begin{array}{l}\text { The leaves of J.adhataoda, C.sinensis and F. vulgare }(10 \mathrm{~g} \\
\text { each) are boiled in water at night Decoction is filtered and } \\
\text { used early in morning }\end{array}$ & $\begin{array}{l}\text { Stomachache, Bleeding } \\
\text { dysentery }\end{array}$ & $\begin{array}{l}\text { Orakzai, Gujar } \\
\text { Khan, Cholistan }\end{array}$ & $\begin{array}{l}\text { Hayat et al.,2008; Ullah et } \\
\text { al.,2010; Jan et al.,2008 }\end{array}$ \\
\hline $\begin{array}{l}\text { Lallemantia royleana } \\
\text { Benth. Labiatae }\end{array}$ & $\begin{array}{l}\text { Tukm-e- } \\
\text { BalangahVi } \\
\text { llanays }\end{array}$ & A & $\mathrm{H}$ & $\mathrm{S}$ & $\begin{array}{l}\text { Seeds are soaked in water overnight make infusion. Eat } \\
\text { seeds up to } 2-5 \text { gram for carminative. }\end{array}$ & $\begin{array}{l}\text { Stomach troubles } \\
\text { Carminative }\end{array}$ & Mandi Bahaudin & $\begin{array}{l}\text { Hayat et al.,2008; Adnan et } \\
\text { al.,2014 }\end{array}$ \\
\hline $\begin{array}{l}\text { Lawsonia inermis Lam. } \\
\text { Lythraceae }\end{array}$ & $\begin{array}{l}\text { Revandchin } \\
\text { i }\end{array}$ & $\mathrm{P}$ & $\mathrm{H}$ & $\mathrm{L}, \mathrm{S}$ & Leaves and Seeds are used & Dysentry & Bannu & Hussain et al.,2008 \\
\hline $\begin{array}{l}\text { Lepidium draba } \mathrm{L} . \\
\text { Brassicaceae }\end{array}$ & Bashka & A & $\mathrm{H}$ & WP & Fresh plant is used & Stomachache & Dir, kohistan valley & Farooq et al.,2012 \\
\hline $\begin{array}{l}\text { Leptadenia } \\
\text { pyrotechnica } \text { Forssk. } \\
\text { Asclepiadaceae }\end{array}$ & Markh & A & $\mathrm{S}$ & WP & Powder of whole plant used & Carminative & $\begin{array}{l}\text { Bugrote valleys } \\
\text { gilgit }\end{array}$ & Safa et al.,2012 \\
\hline $\begin{array}{l}\text { Lycopersicon } \\
\text { esculentum Mill. } \\
\text { Solanaceae }\end{array}$ & Tamatar, & $\mathrm{A} / \mathrm{P}$ & $\mathrm{H}$ & Fr & Fresh juice of riped fruit is used to reduce acidity & Stomach acidity & Lodheran, Punjab & Hayat et al., 2008 \\
\hline $\begin{array}{l}\text { Malva neglecta Wall. } \\
\text { Malvaceae }\end{array}$ & Panerak & $\mathrm{P}$ & $\mathrm{H}$ & $\mathrm{L}, \mathrm{R}$ & $\begin{array}{l}\text { Decoction of leaves is used as a laxative and remedy for } \\
\text { tape worm. }\end{array}$ & $\begin{array}{l}\text { Laxative, } \\
\text { Worms }\end{array}$ & Lakki Marwat & $\begin{array}{l}\text { Farooq et al.,2012; Hassan et } \\
\text { al.,2014 }\end{array}$ \\
\hline $\begin{array}{l}\text { Melia azedarach } \\
\text { L.Meliaceae }\end{array}$ & $\begin{array}{l}\text { Dharek/ } \\
\text { Bakain }\end{array}$ & $\mathrm{P}$ & $\mathrm{T}$ & $\mathrm{Wp}$ & $\begin{array}{l}200 \mathrm{~g} \text { of fresh leaves, twigs and fruits are crushed for gas } \\
\text { troubles and indigestion. }\end{array}$ & Gas trouble, Indigestion & Lakki Marwat & $\begin{array}{l}\text { Manan et al.,2007; Khan et } \\
\text { al.,2012 }\end{array}$ \\
\hline $\begin{array}{l}\text { Mentha arvensis } \mathrm{L} . \\
\text { Labiatae }\end{array}$ & Podina & $\mathrm{P}$ & $\mathrm{H}$ & $\mathrm{L}$ & $\begin{array}{l}\text { Dried leaves are taken with curd to control dysentery, to } \\
\text { stop vomiting and nausea, cholera }\end{array}$ & $\begin{array}{l}\text { Dysentry,Vomiting } \\
\text { Indigestion,Cholera }\end{array}$ & $\begin{array}{l}\text { Punjab, } \\
\text { Balochistan }\end{array}$ & $\begin{array}{l}\text { Shah \& Khan., 2006; Begum } \\
\text { et al.,2014 }\end{array}$ \\
\hline $\begin{array}{l}\text { Mentha longifolia } \mathrm{L} \text {. } \\
\text { Labiatae }\end{array}$ & $\begin{array}{l}\text { Enaley, } \\
\text { Ben }\end{array}$ & $\mathrm{P}$ & $\mathrm{H}$ & $\begin{array}{l}\mathrm{L}, \mathrm{S}, \\
\mathrm{R}\end{array}$ & $\begin{array}{l}\text { Fresh leaves and shoots as anti-diarrhoeal. Paste of leaves } \\
\text { and tender shoots with garlic and little salt is given trice a } \\
\text { day }\end{array}$ & $\begin{array}{l}\text { Dysentery,Diarrhea, } \\
\text { Stomachache, Vomiting }\end{array}$ & $\begin{array}{l}\text { Jhelum, Mirpur, } \\
\text { AJK }\end{array}$ & $\begin{array}{l}\text { Hassan et al.,2014; } \\
\text { Barkattullah et al.,2009; } \\
\text { Hussain et al.,2008 }\end{array}$ \\
\hline $\begin{array}{l}\text { Mentha royleana Benth. } \\
\text { Labiatae }\end{array}$ & $\begin{array}{l}\text { khwaa } \\
\text { bootei }\end{array}$ & A & $\mathrm{H}$ & $\mathrm{Wp}$ & $\begin{array}{l}\text { Leaves are mixed in green teas and are used in vomiting, as } \\
\text { cooling agent. Decoction is used as carminative. }\end{array}$ & $\begin{array}{l}\text { Vomiting, Gas trouble, } \\
\text { Carminative }\end{array}$ & Mandi Bahaudin & $\begin{array}{l}\text { Khan et al.,2013; Noor et } \\
\text { al.,2012 }\end{array}$ \\
\hline $\begin{array}{l}\text { Mentha Spicata } \mathrm{L} . \\
\text { Labiatae }\end{array}$ & Poodna & A & $\mathrm{H}$ & $\mathrm{L}$ & Leaves decoction taken orally & Carminative, Diarrhea & $\begin{array}{l}\text { Balochistan, Kurd } \\
\text { Sharif }\end{array}$ & Mahmood et al.,2013 \\
\hline $\begin{array}{l}\text { Mentha sylvestris } \mathrm{L} \text {. } \\
\text { Labiatae }\end{array}$ & $\begin{array}{l}\text { Lewanii } \\
\text { Vellana }\end{array}$ & A & $\mathrm{H}$ & $\mathrm{L}$ & Decoction of the plant is used & $\begin{array}{l}\text { Carminative, } \\
\text { Nausea,Vomiting }\end{array}$ & $\begin{array}{l}\text { Lakki Marwat, } \\
\text { Jhelum }\end{array}$ & Farooq et al.,2012 \\
\hline $\begin{array}{l}\text { Mentha virdis L. } \\
\text { Labiatae }\end{array}$ & Podina & $\mathrm{P}$ & $\mathrm{H}$ & WP & Decoction is used.Dry leaves powder used as carminative. & $\begin{array}{l}\text { Flatulence, Indigestion, } \\
\text { Stomachache } \\
\text { Carminative }\end{array}$ & Sialkot & $\begin{array}{l}\text { Mussarat et al.,2014; Farooq } \\
\text { et al.,2012; Hussain et } \\
\text { al.,2012 }\end{array}$ \\
\hline $\begin{array}{l}\text { Momordica balsamica } \\
\text { L. Cucurbitaceae }\end{array}$ & $\begin{array}{l}\text { Jangli } \\
\text { Kareel }\end{array}$ & $\mathrm{P}$ & $\mathrm{T}$ & $\mathrm{F}$ & $\begin{array}{l}\text { Fruit is used to treat gas trouble and constipation. By using } \\
\text { its extract to kill the abdominal worms. }\end{array}$ & $\begin{array}{l}\text { Constipation } \\
\text { Abdominal worms }\end{array}$ & Attock & $\begin{array}{l}\text { Manan et al.,2007; Shaheen } \\
\text { et al.,2012 }\end{array}$ \\
\hline $\begin{array}{l}\text { Momordica charantia } \\
\text { L. Cucurbitaceae }\end{array}$ & Karela & A & $\mathrm{S}$ & $\mathrm{F}$ & Fruit is used to treat intestinal worms and dysentry. & Worms, Dysentry & Malakand & Shaheen et al.,2012 \\
\hline
\end{tabular}


Adnan et al., Afr J Tradit Complement Altern Med. (2015) 12(5):99-117

http://dx.doi.org/10.4314/ajtcam.v12i5.15

\begin{tabular}{|c|c|c|c|c|c|c|c|c|}
\hline $\begin{array}{l}\text { Morus nigra } \mathrm{L} . \\
\text { Moraceae }\end{array}$ & Kala Toot & A & $\mathrm{T}$ & $\mathrm{L}$ & The decoction of young leaves is used & Diarrhea & Mansehra & Hussain et al.,2010 \\
\hline $\begin{array}{l}\text { Mukia maderaspatana } \\
\text { L. Cucurbitaceae }\end{array}$ & Khotilal & A & $\mathrm{H}$ & $\mathrm{R}$ & The decoction of root is useful & Flatulence & Attock & Qureshi et al.,2010 \\
\hline $\begin{array}{l}\text { Murraya exotica L. } \\
\text { Rutaceae }\end{array}$ & Marva & A & $\mathrm{S}$ & $\mathrm{R}, \mathrm{L}$ & Roots and leaves extract with sugar or salt is useful & $\begin{array}{l}\text { Helminths,DiarrheaCar } \\
\text { minative Dysentery }\end{array}$ & Humzoni, Nwa & Hussain et al.,2010 \\
\hline $\begin{array}{l}\text { Nasturtium offficinale } \\
\text { R.Br. Brassicaceae }\end{array}$ & Termera & $\mathrm{P}$ & $\mathrm{H}$ & $\mathrm{L}$ & $\begin{array}{l}\text { Fresh leaves are cooked as vegetables and taken orally for } \\
\text { constipation. Local hakims use it in tablets, for } \\
\text { stomachache. }\end{array}$ & $\begin{array}{l}\text { Constipation } \\
\text { Stomachache }\end{array}$ & $\begin{array}{l}\text { Dir, kohistan valley, } \\
\text { D.I.Khan }\end{array}$ & $\begin{array}{l}\text { Abbasi et al.,2013; } \\
\text { Barkattullah et al., } 2009\end{array}$ \\
\hline $\begin{array}{l}\text { Ocimum bacilum } \mathrm{L} \text {. } \\
\text { Labiatae }\end{array}$ & Gusmaly & $\mathrm{P}$ & $\mathrm{H}$ & $\mathrm{S}, \mathrm{L}$ & $\begin{array}{l}\text { Mucilaginous seeds are used in diarrhea and chronic } \\
\text { dysentery. Decoction of leaves is useful in diarrhoea and } \\
\text { tonic for stomach. Leaves mixed with mint and little for } \\
\text { stomachache and vomiting. }\end{array}$ & $\begin{array}{l}\text { Diarrhea } \\
\text { Dysentry } \\
\text { Stomachache Vomiting }\end{array}$ & Chitral & $\begin{array}{l}\text { Hayat et al.,2008; Ballabh \& } \\
\text { Chuarasia., 2009; Shadangi et } \\
\text { al.,2012; Hussain et al.,2008 }\end{array}$ \\
\hline $\begin{array}{l}\text { Olea ferruginia } \\
\text { Royle.Oleaceae }\end{array}$ & Kahu/ kohi & $\mathrm{P}$ & $\mathrm{H}$ & $\mathrm{F}$ & Extract of fruits is given orally for 5-6 days & Indigestion & $\begin{array}{l}\text { Bumburet Valley } \\
\text { Chitral }\end{array}$ & $\begin{array}{l}\text { Rauf et al.,2012; Khan et } \\
\text { al.,2012 }\end{array}$ \\
\hline $\begin{array}{l}\text { Opuntia dillenii Haw. } \\
\text { Cactaceae }\end{array}$ & Kunda thur & $\mathrm{P}$ & $\mathrm{S}$ & $\mathrm{F}$ & $\begin{array}{l}\text { Decoction of fruits with sugar and kept in a bottle and } 2 \\
\text { tablespoon of juice used twice a day for } 21 \text { days }\end{array}$ & $\begin{array}{l}\text { Piles } \\
\text { Inflammation }\end{array}$ & D. I. Khan & Rajasab \& Isaq., 2007 \\
\hline $\begin{array}{l}\text { Opuntia monacantha } \\
\text { Haw. Cactaceae }\end{array}$ & Chnutarthar & $\mathrm{P}$ & $\mathrm{S}$ & WP & Used in powder form & $\begin{array}{l}\text { Laxation, Stomachache } \\
\text { Carminative }\end{array}$ & D.I.Khan & Ahmad., 2007 \\
\hline $\begin{array}{l}\text { Origanum vulgare } \mathrm{L} . \\
\text { Labiatae }\end{array}$ & $\begin{array}{l}\text { Jangli } \\
\text { Ajwain }\end{array}$ & $\mathrm{P}$ & $\mathrm{H}$ & Wp & $\begin{array}{l}\text { Leaves extract and Whole plant Powder mixed with milk is } \\
\text { taken }\end{array}$ & $\begin{array}{l}\text { Stomachache } \\
\text { Colic, Diarrhea, Ulcers, } \\
\text { Vomiting }\end{array}$ & Kurd Sharif, Karak & $\begin{array}{l}\text { Murad et al.,2012; Bhatt \& } \\
\text { Negi, 2006; Arshad \& } \\
\text { Ahmad., 2004 }\end{array}$ \\
\hline $\begin{array}{l}\text { Oroxylum indicum } \\
\text { Linn. Bignoniaceae }\end{array}$ & $\begin{array}{l}\text { Talwar } \\
\text { phalli }\end{array}$ & $\mathrm{P}$ & $\mathrm{T}$ & $\begin{array}{l}\text { F, St, } \\
\text { B }\end{array}$ & $\begin{array}{l}\text { Fruits and stem are dried and powdered make pills and } \\
\text { taken thrice daily for } 1-2 \text { months }\end{array}$ & $\begin{array}{l}\text { Dysentry } \\
\text { Piles }\end{array}$ & North Waziristan & $\begin{array}{l}\text { Punjani., 2002; Hossan et } \\
\text { al.,2009 }\end{array}$ \\
\hline $\begin{array}{l}\text { Otostegia limbata } \\
\text { Boiss. Labiatae }\end{array}$ & Chitta jand & A & $\mathrm{T}$ & $\mathrm{L}$ & Leaves are crushed and used & Acidity & Jhelum & Rauf et al.,2012 \\
\hline $\begin{array}{l}\text { Oxalis corniculata } \mathrm{L} . \\
\text { Oxalidaceae }\end{array}$ & Marveeza & $\mathrm{P}$ & $\mathrm{H}$ & WP & Whole plant infusion use orally & $\begin{array}{l}\text { Diarrhea,Dysentery } \\
\text { Constipation } \\
\text { Stomachache }\end{array}$ & $\begin{array}{l}\text { Malakand Valley } \\
\text { Dist Dir (Lower) }\end{array}$ & Murad et al., 2012 \\
\hline $\begin{array}{l}\text { Parthenium } \\
\text { hysterophorus } \mathrm{L} . \\
\text { Asteraceae }\end{array}$ & Thandi boti & A & $\mathrm{H}$ & WP & Decoction of plant is used & Dysentry & $\begin{array}{l}\text { Lahore-Islamabad } \\
\text { Motoway }\end{array}$ & Mahmood et al.,2011 \\
\hline $\begin{array}{l}\text { Peganum harmala } \mathrm{L} . \\
\text { Zygophyllaceae }\end{array}$ & $\begin{array}{l}\text { KisankoorS } \\
\text { ponda/Spell } \\
\text { anii }\end{array}$ & Pn & $\mathrm{S}$ & WP & $\begin{array}{l}1 / 2 \mathrm{~kg} \text { fresh leaves ground with salt; paste is orally } \\
\text { administered for 5- } 6 \text { days for gastric problems. Plant soaked } \\
\text { in water and infusion is used for abdominal pain. Powered } \\
\text { seeds are given twice a day in small dose for stomachache } \\
\text { for 2-3 days. }\end{array}$ & $\begin{array}{l}\text { Abdominal pain, } \\
\text { Gastric problems } \\
\text { Vomiting } \\
\text { Abdominal pain } \\
\text { Stomachache }\end{array}$ & Gallyat & $\begin{array}{l}\text { Hayat et al.,2008; Ballabh \& } \\
\text { Chaurasia., 2009; Tareen et } \\
\text { al.,2010; Noor et al.,2012; } \\
\text { Sultana et al.,2006 }\end{array}$ \\
\hline $\begin{array}{l}\text { Phyllanthus emblica L. } \\
\text { Euphorbiaceae }\end{array}$ & Amla & A & $\mathrm{T}$ & $\mathrm{B}, \mathrm{F}$ & Bark and fruit decoction use orally & Inflammation Dysentry & $\begin{array}{l}\text { Makerwal \& Gulla } \\
\text { Khel }\end{array}$ & $\begin{array}{l}\text { Abbasi et al.,2013; Hussain et } \\
\text { al.,2008 }\end{array}$ \\
\hline $\begin{array}{l}\text { Pimpinella stewartii } \\
\text { Dunn. Umbelliferae }\end{array}$ & & $\mathrm{P}$ & S & F & Fruit is eaten & Carminative & Chitral & $\begin{array}{l}\text { Afzal et al.,2009; Awan et } \\
\text { al.,2001 }\end{array}$ \\
\hline $\begin{array}{l}\text { Plectranthus rugosus } \\
\text { Wall. Lamiaceae }\end{array}$ & Spairkay & A & $S$ & Sh & Decoction and powder is used & Inflammation & Jhelum & Alamgeer et al.,2013 \\
\hline
\end{tabular}


Adnan et al., Afr J Tradit Complement Altern Med. (2015) 12(5):99-117

http://dx.doi.org/10.4314/ajtcam.v12i5.15

\begin{tabular}{|c|c|c|c|c|c|c|c|c|}
\hline $\begin{array}{l}\text { Pongamia pinnata L. } \\
\text { Papilionaceae }\end{array}$ & Sukh Chain & $\mathrm{P}$ & $\mathrm{T}$ & $\mathrm{L}$ & Powder is taken orally & Gas trouble & $\begin{array}{l}\text { Lahore_Islamabad } \\
\text { motorway }\end{array}$ & Mahmood et al.,2013 \\
\hline $\begin{array}{l}\text { Prunus armeniaca } \mathrm{L} \text {. } \\
\text { Rosaceae }\end{array}$ & Zule & $\mathrm{P}$ & $\mathrm{T}$ & $\mathrm{F}$ & The decoction of the dried fruits is used & Laxative and Purgative & Balochistan & Sher et al.,2009 \\
\hline $\begin{array}{l}\text { Psidium guajava } \mathrm{L} \text {. } \\
\text { Myrtaceae }\end{array}$ & $\begin{array}{l}\text { Amrood, } \\
\text { Guava }\end{array}$ & $\mathrm{P}$ & $\mathrm{T}$ & Fr, L & $\begin{array}{l}\text { Whole fruit is eaten simply as a purgative. Fresh leaves and } \\
\text { paste of tender twig with cold water is taken orally for } \\
\text { diarrhea }\end{array}$ & $\begin{array}{l}\text { Purgative, Blood } \\
\text { dysentery } \\
\text { Diarrhea }\end{array}$ & Karak & $\begin{array}{l}\text { Hussain et al.,2010; Shadangi } \\
\text { et al.,2012; Adnan et al.,2014 }\end{array}$ \\
\hline $\begin{array}{l}\text { Ricinus communis } \mathrm{L} \text {. } \\
\text { Euphorbiaceae }\end{array}$ & $\begin{array}{l}\text { Hernoli, } \\
\text { Castor oil }\end{array}$ & $\mathrm{P}$ & $\mathrm{S}$ & $\mathrm{S}$ & Seed oil is used & Constipation & $\begin{array}{l}\text { Swat, Lahore- } \\
\text { Islamabad } \\
\text { motorway }\end{array}$ & $\begin{array}{l}\text { Abbas et al.,2013; Hussain et } \\
\text { al.,2010; Arshad et al.,2011 }\end{array}$ \\
\hline $\begin{array}{l}\text { Rosa damascena Mill. } \\
\text { Rosaceae }\end{array}$ & Gulab. & $\mathrm{P}$ & $\mathrm{S}$ & $\mathrm{Fl}$ & Powdered flowers with Quercus fruit is used & $\begin{array}{l}\text { Hemorrhoid and } \\
\text { Ulcer }\end{array}$ & Punjab & $\begin{array}{l}\text { Iqbal et al.,2011; Amir et } \\
\text { al.,2013 }\end{array}$ \\
\hline $\begin{array}{l}\text { Rosa indica } \mathrm{L} . \\
\text { Rosaceae }\end{array}$ & Gulab & $P$ & $\mathrm{~S}$ & $\mathrm{Fl}$ & $\begin{array}{l}\text { Petals of flowers and sugar are put in jar for two to three } \\
\text { days and the product (gulkand) is taken for constipation and } \\
\text { abdominal pain. }\end{array}$ & $\begin{array}{l}\text { Constipation and } \\
\text { Abdominal pain }\end{array}$ & Punjab, D. I. Khan & $\begin{array}{l}\text { Khan et al.,2013; Sultana et } \\
\text { al.,2006; Mussarat et al.,2014 }\end{array}$ \\
\hline $\begin{array}{l}\text { Rosa webbiana Wall. } \\
\text { Rosaceae }\end{array}$ & Palwari & B & $\mathrm{S}$ & $\mathrm{F}, \mathrm{Fl}$ & Flower and fruit juice is consumed to cure stomach pain. & Stomachache & Balochistan & $\begin{array}{l}\text { Singh et al.,2012; Wazir et } \\
\text { al.,2004 }\end{array}$ \\
\hline Salix alba L. Salicaceae & Walla & $\mathrm{P}$ & $\mathrm{T}$ & $\mathrm{B}, \mathrm{L}$ & Decoction is used & Diarrhea, Dysentry & $\begin{array}{l}\text { Rawlakot-Azad } \\
\text { jamu \& Kashmir }\end{array}$ & $\begin{array}{l}\text { Mardaninejad et al.,2013; } \\
\text { Qureshi et al.,2006 }\end{array}$ \\
\hline $\begin{array}{l}\text { Salvadora oleoides } \\
\text { Decne. Salvadoraceae }\end{array}$ & $\begin{array}{l}\text { Banbakhara } \\
\text {,Jhal }\end{array}$ & $\mathrm{B}, \mathrm{A}$ & $\mathrm{S}$ & $\begin{array}{l}\text { L, F, } \\
\text { St }\end{array}$ & $\begin{array}{l}\text { Decoction of leaf, fruit and stem used. The unripe fruits are } \\
\text { pickled and used to treat constipation. }\end{array}$ & $\begin{array}{l}\text { Diarrhea, Purgative } \\
\text { Constipation }\end{array}$ & $\begin{array}{l}\text { Leepa Valley } \\
\text { Muzaffarabad, } \\
\text { Gilgit }\end{array}$ & Shaheen et al.,2012 \\
\hline $\begin{array}{l}\text { Salvadora persicaL. } \\
\text { Salvadoraceae }\end{array}$ & Peelu & $\mathrm{B} / \mathrm{P}$ & $\mathrm{S}$ & $\mathrm{S}, \mathrm{L}$ & Decoction is used & $\begin{array}{l}\text { Constipation } \\
\text { Stomachache }\end{array}$ & $\begin{array}{l}\text { Jalalpur Jattan, } \\
\text { Gujrat }\end{array}$ & Tareen et al.,2010 \\
\hline $\begin{array}{l}\text { Salvia cabulica Bth. } \\
\text { Labiatae }\end{array}$ & Matetav & A & $\mathrm{H}$ & $\mathrm{L}$ & Leaves are soaked in water and the decoction is used & Stomachache & Jhelum & Tareen et al.,2010 \\
\hline $\begin{array}{l}\text { Sisymbrium irio } \\
\text { L.Brassicaceae }\end{array}$ & $\begin{array}{l}\text { Jangli } \\
\text { sarsoo }\end{array}$ & $\mathrm{A}$ & $\mathrm{H}$ & $\mathrm{S}$ & Crushed seeds are used & $\begin{array}{l}\text { Dysentery, Diarrhea } \\
\text { Vomiting }\end{array}$ & Dir, kohistan valley & $\begin{array}{l}\text { Murad et al.,2013; Hussain et } \\
\text { al.,2008 }\end{array}$ \\
\hline $\begin{array}{l}\text { Solanum miniatum } \\
\text { Beruh. Solanaceae }\end{array}$ & Peelak. & $\mathrm{P}$ & $\mathrm{H}$ & $\mathrm{L}$ & Decoction takentwice a day for 6-7 days & Abdominal swellowing & $\begin{array}{l}\text { Poonch Valley Azad } \\
\text { Kashmir }\end{array}$ & $\begin{array}{l}\text { Abbas et al.,2013; Iqbal et } \\
\text { al.,2011 }\end{array}$ \\
\hline $\begin{array}{l}\text { Solanum nigrum } \mathrm{L} . \\
\text { Solanaceae }\end{array}$ & Mako & $\mathrm{A}$ & $\mathrm{H}$ & WP & $\begin{array}{l}\text { Whole plant infusion use orally for diarrhea.Ripe fruits are } \\
\text { directly given orally for constipation. } \\
\text { Plant juice is used for dysentery. }\end{array}$ & $\begin{array}{l}\text { Diarrhea } \\
\text { Constipation } \\
\text { Dysentry } \\
\end{array}$ & D. I. Khan Karak & $\begin{array}{l}\text { Mussarat et al.,2014; Murad et } \\
\text { al.,2012 }\end{array}$ \\
\hline $\begin{array}{l}\text { Solanum surattense } \\
\text { Burm. Solanaceae }\end{array}$ & $\begin{array}{l}\text { Kanderi, } \\
\text { Mahukeri }\end{array}$ & A & $\mathrm{S}$ & WP & $\begin{array}{l}\text { Pills of plant use for vomiting. } 200 \mathrm{~g} \text { fresh plant is boiled } \\
\text { with black pepper and salt and given orally }\end{array}$ & $\begin{array}{l}\text { Stomachache } \\
\text { Vomiting } \\
\text { Indigestion }\end{array}$ & $\begin{array}{l}\text { Poonch Valley Azad } \\
\text { Kashmir }\end{array}$ & $\begin{array}{l}\text { Abbas et al.,2013; Murad et } \\
\text { al.,2012 }\end{array}$ \\
\hline $\begin{array}{l}\text { Spinacea oleracea } \mathrm{L} \text {. } \\
\text { Chenopodiaceae }\end{array}$ & Paluk & $\mathrm{A}$ & $\mathrm{H}$ & $\mathrm{L}$ & Boil 5-8 leaves in water and take orally & Stomach calming & Khushab & $\begin{array}{l}\text { Adnan et al.,2014; Akhter et } \\
\text { al.,2013 }\end{array}$ \\
\hline $\begin{array}{l}\text { Swertia chirata } \mathrm{L} . \\
\text { Gentianaceae }\end{array}$ & Chiraita & B & $\mathrm{H}$ & WP & $\begin{array}{l}\text { Aerial parts are dried and crushed to use for stomach trouble } \\
\text { and digestive disorders. Whole plant decoction is taken } \\
\text { orally for gastro-entritis. }\end{array}$ & $\begin{array}{l}\text { Indigestion } \\
\text { Gastroentritis }\end{array}$ & Kala Bagh & $\begin{array}{l}\text { Arshad \& Ahmad., 2004; } \\
\text { Ningombam et al.,2014 }\end{array}$ \\
\hline $\begin{array}{l}\text { Tecomella undulate } \\
\text { Bignoniaceae }\end{array}$ & Purpak & A & $\mathrm{H}$ & $\mathrm{B}$ & Decoction of the bark is used & $\begin{array}{l}\text { Constipation } \\
\text { Stomachache }\end{array}$ & Mansehra & $\begin{array}{l}\text { Tareen et al.,2010; Safa et } \\
\text { al.,2012 }\end{array}$ \\
\hline
\end{tabular}


Adnan et al., Afr J Tradit Complement Altern Med. (2015) 12(5):99-117

http://dx.doi.org/10.4314/ajtcam.v12i5.15

\begin{tabular}{|c|c|c|c|c|c|c|c|c|}
\hline $\begin{array}{l}\text { Tephrosia lupinifolia } \\
\text { DC. Papilionaceae }\end{array}$ & Fish Poison & A & $\mathrm{H}$ & $\begin{array}{l}\text { R, L, } \\
\text { St, B }\end{array}$ & Powder of plant parts & Stomachache, Diarrhea & Southern punjab & $\begin{array}{l}\text { Hussain et al.,2010; Arshad et } \\
\text { al.,2011 }\end{array}$ \\
\hline $\begin{array}{l}\text { Terminalia chebula } \\
\text { Retz. Combretaceae }\end{array}$ & Harar & B & $\mathrm{T}$ & F & $\begin{array}{l}\text { Powdered fruit is mixed with water or cow's or goat's milk } \\
\text { and taken }\end{array}$ & Stomachache & Dir Kohistan Valley & Ignacimuthu et al.,2006 \\
\hline $\begin{array}{l}\text { Thymus linearis Benth. } \\
\text { Labiatae }\end{array}$ & Sew & A & $\mathrm{H}$ & WP & Whole plant is boiled in water and used & $\begin{array}{l}\text { Indigestion } \\
\text { Carminative }\end{array}$ & Sialkot & $\begin{array}{l}\text { Ali \& Qaiser., 2009; Noor et } \\
\text { al.,2012; Humayun., } 2007\end{array}$ \\
\hline $\begin{array}{l}\text { Thymus serphyllum } \mathrm{L} . \\
\text { Labiatae }\end{array}$ & Pangdum & $\mathrm{P}$ & $\mathrm{H}$ & WP & $\begin{array}{l}\text { Seeds are grinded and one spoon used daily with water. } \\
\text { Powder of flowers is mixed in "Gur" and given as } \\
\text { vermicide. }\end{array}$ & $\begin{array}{l}\text { Abdominal pain, } \\
\text { internal wound } \\
\text { Worms }\end{array}$ & Mirpur, AJK & $\begin{array}{l}\text { Shah \& Khan., 2006; Farooq } \\
\text { et al.,2012; Qureshi et } \\
\text { al.,2007 }\end{array}$ \\
\hline $\begin{array}{l}\text { Tordylium nodosum } \mathrm{L} \\
\text { Apiaceae }\end{array}$ & Hoso beta & A & $\mathrm{H}$ & $\mathrm{R}$ & The juice of the root is used & Indigestion & Bannu & Khan et al.,2013 \\
\hline $\begin{array}{l}\text { Trachyspermum ammi } \\
\text { L. Umbelliferae }\end{array}$ & Ajwain & A & $\mathrm{T}$ & $\mathrm{S}$ & Seeds are eaten before meal with water or milk & $\begin{array}{l}\text { Stomachache, Digestive } \\
\text { disorder }\end{array}$ & $\begin{array}{l}\text { Valley Alladand } \\
\text { Dehri Malakand }\end{array}$ & $\begin{array}{l}\text { Humayun et al.,2006; Iqbal et } \\
\text { al.,2011 }\end{array}$ \\
\hline $\begin{array}{l}\text { Trachyspermum ammi } \\
\text { L.Umbelliferae }\end{array}$ & Ajwain & $\mathrm{P}$ & $\mathrm{H}$ & $\mathrm{S}$ & Seeds are taken with little salt & Gas trouble & Chitral & Sultana et al.,2006 \\
\hline $\begin{array}{l}\text { Tribulus terrestris } \mathrm{L} . \\
\text { Zygophyllaceae }\end{array}$ & $\begin{array}{l}\text { Bakhra, } \\
\text { spalani }\end{array}$ & A & $\mathrm{H}$ & $\mathrm{F}$ & The powder of fruits is mixed in water and taken & Diarrhea & Gallyat & $\begin{array}{l}\text { Hussain et al.,2008; Prusti \& } \\
\text { Behera., } 2007\end{array}$ \\
\hline $\begin{array}{l}\text { Typha angustata B \& } \\
\text { C.Typhaceae }\end{array}$ & Lokhay & $\mathrm{P}$ & $\mathrm{H}$ & $\mathrm{L}$ & Dry the leaves are grind and cooked & Gas trouble & $\begin{array}{l}\text { Booni Valley, } \\
\text { Chitral }\end{array}$ & Adnan et al.,2014 \\
\hline $\begin{array}{l}\text { Verbiscum thepsus } \mathrm{L} . \\
\text { Schryophylaceae }\end{array}$ & Ghadi Kan & A & $\mathrm{H}$ & WP & Tea of leaves is used & $\begin{array}{l}\text { Dysentry } \\
\text { Diarrhea }\end{array}$ & $\begin{array}{l}\text { Jalalpur Jattan, } \\
\text { Gujrat }\end{array}$ & $\begin{array}{l}\text { Abbas et al.,2013; Noor et } \\
\text { al.,2012 }\end{array}$ \\
\hline $\begin{array}{l}\text { Veronica anagallis } \mathrm{L} . \\
\text { Plantaginaceae }\end{array}$ & Hazar booti & A & $\mathrm{H}$ & WP & Whole plant is used & Stomach upset & $\begin{array}{l}\text { Jalalpur Jattan, } \\
\text { Gujrat }\end{array}$ & Gulshan et al.,2012 \\
\hline $\begin{array}{l}\text { Viola odorata } \mathrm{L} . \\
\text { Violaceae }\end{array}$ & Banafsha & $\mathrm{P}$ & $\mathrm{H}$ & $\mathrm{R}$ & Oil is used & Abdominal pain & Malakand & Chaudary et al.,2013 \\
\hline $\begin{array}{l}\text { Viola serpensWall. } \\
\text { Violaceae }\end{array}$ & Binafsha & $\mathrm{P}$ & $\mathrm{T}$ & $\mathrm{F}, \mathrm{L}$ & One cup of decoction is used three times in a day & Inflammation & Malakand & Begum et al.,2014 \\
\hline $\begin{array}{l}\text { Vitex agnus-castus } \mathrm{L} . \\
\text { Verbenaceae }\end{array}$ & Gowanik & A & $\mathrm{T}$ & $\mathrm{S}$ & Seeds are boiled in water and decoction is used & Stomachache & Malakand & Tareen et al.,2010 \\
\hline $\begin{array}{l}\text { Vitex negundo L. } \\
\text { Verbenaceae }\end{array}$ & Marvandi & $\mathrm{P}$ & $\mathrm{T}$ & $\begin{array}{l}\text { S, L, } \\
\text { R, St, } \\
\text { B }\end{array}$ & $\begin{array}{l}\text { Powder of } T \text {. ammi seeds, } V \text {. negundo dried seeds and table } \\
\text { salt is taken with water twice a day }\end{array}$ & $\begin{array}{l}\text { Cholera, Gas } \\
\text { trouble,Worms, } \\
\text { Carminative }\end{array}$ & Malakand & $\begin{array}{l}\text { Abbasi et al.,2010; Safa et } \\
\text { al.,2012 }\end{array}$ \\
\hline $\begin{array}{l}\text { Withania coagulans } \\
\text { Dunal.Solanaceae }\end{array}$ & $\begin{array}{l}\text { Khapyanga } \\
\text {, Paneer }\end{array}$ & A & $\mathrm{H}$ & $\mathrm{F}$ & $\begin{array}{l}\text { Fruit is used for dyspepsia and flatulence.Five to six fruits is } \\
\text { taken with water like tablet for abdominal pain. }\end{array}$ & $\begin{array}{l}\text { Dyspepsia } \\
\text { Abdominal pain }\end{array}$ & $\begin{array}{l}\text { Swat, Karak, D. I. } \\
\text { Khan }\end{array}$ & $\begin{array}{l}\text { Murad et al.,2013; Mussarat et } \\
\text { al.,2014; Farooq et al.,2012 }\end{array}$ \\
\hline $\begin{array}{l}\text { Withania somnifera } \mathrm{L} \text {. } \\
\text { Solanaceae }\end{array}$ & Shapianga & A & $\mathrm{H}$ & F, L, S & $\begin{array}{l}\text { Flower and leaf decoction use in treatment of Anthelmintic. } \\
\text { Put } 2-3 \text { seeds or fruits into the water and then eat }\end{array}$ & $\begin{array}{l}\text { Helminths } \\
\text { Carminative }\end{array}$ & Bannu & Adnan et al.,2014 \\
\hline $\begin{array}{l}\text { Xanthium strumarium } \\
\text { L. Asteraceae }\end{array}$ & $\begin{array}{l}\text { Chhota } \\
\text { Dhatura }\end{array}$ & A & $\mathrm{H}$ & $\mathrm{L}, \mathrm{R}$ & Decoction is used. & Laxative & $\begin{array}{l}\text { Khirthar National } \\
\text { Park, Rawalpindi }\end{array}$ & $\begin{array}{l}\text { Hussain et al.,2010; Waris et } \\
\text { al.,2012; Arshad et al.,2011 }\end{array}$ \\
\hline $\begin{array}{l}\text { Zanthoxylum armatum } \\
\text { L. Rutaceae }\end{array}$ & Gojri & $\mathrm{P} / \mathrm{A}$ & S & $\mathrm{L}, \mathrm{S}, \mathrm{F}$ & $50 \mathrm{~g}$ seed are orally administered with flour & Indigestion & Humzoni Nwa & Abbas et al.,2013 \\
\hline $\begin{array}{l}\text { Zataria multiflora } \\
\text { Boiss. Labiatae }\end{array}$ & $\begin{array}{l}\text { Thrushnagh } \\
\text { ooli }\end{array}$ & $\mathrm{P}$ & $\mathrm{S}$ & $\mathrm{L}, \mathrm{Sh}$ & Powder is used & Diarrhea, Stomachache & Sialkot & $\begin{array}{l}\text { Ali \& Qaiser., 2009; Safa et } \\
\text { al.,2012 }\end{array}$ \\
\hline
\end{tabular}

Boiss. Labiatae

Thrushnagh

Key: Aerial part= AP, Bark= B, Flower bud= FB, Flower= F, Fruit= Fr, Latex= La, Leaves= L, Pulp= P, Resin= Re, Rhizome= Rh, Root= R, Sap= Sp, Seed= S, Stem= St, Twig= Tw, Whole al.,2012 plant $=$ WP, Wood $=$ W, Tree $=$ T, Herb $=$ H, Shrub $=$ S, Annual= A, Biannual $=$ B, Perennial $=$ P

112 
Adnan et al., Afr J Tradit Complement Altern Med. (2015) 12(5):99-117

http://dx.doi.org/10.4314/ajtcam.v12i5.15

Table 2: Ecological status of plant species.

\begin{tabular}{|c|c|c|c|c|}
\hline Plants & Wild/ Cultivated & Endemic & Ecological status & Refrences \\
\hline Acacia nilotica & Cultivated & Endemic & Endangered & Ahmad et al., 2012; Abbasi et al., 2010 \\
\hline Achillea millefolium & & & Vulnerable & Khan., 2011 \\
\hline Acorus calamus & & & Endangered & Humayun et al., 2006 \\
\hline Aesculus indica & & & Endangered & Khan., 2011 \\
\hline Ajuga bracteosa & & Endemic & Threatened & Qamar et al., 2010; Akhtar et al., 2013 \\
\hline Albizia lebbeck & Wild & & Endangered & Ahmad., 2007; Ahmad et al., 2012 \\
\hline Allium sativum & Cultivated & & Protected & Hussain et al., 2012; Ahmad et al., 2012 \\
\hline Barberis lyceum & & Endemic & Threatened & Qamar et al., 2010; Akhtar et al., 2013 \\
\hline Bergenia ciliate & Wild & & Threatened & Abbas et al., 2013; Humayun et al., 2006; Qamar et al., 2010 \\
\hline Bunium persicum & & & Critically endangered & Khan., 2011 \\
\hline Cannabis sativa & Wild & Endemic & Proteced & Abbas et al., 2013; Ahmad et al., 2012; Akhtar et al., 2013 \\
\hline Capparis decidua & Wild & Endemic & Vulnrable & Khan \& Hussain., 2013; Ahmad et al., 2008 \\
\hline Dalbergia sisso & Wild & Endemic & Endngered & Ahmad., 2007; Ahmad et al., 2012; Ahmad et al., 2008 \\
\hline Dodonaea viscosa & Wild & Endemic & Enangered & Ahmad., 2007; Khan \& Hussain., 2013; Ahmad et al., 2008 \\
\hline Elaeagnus angustifolia & Wild & & Vulnerable & Abbas et al., 2013; Khan., 2011 \\
\hline Ephedra gerdiana & Wild & Endemic & Threatened & Abbas et al., 2013; Qamar et al., 2010; Shaheen et al., 2012 \\
\hline Grewia tenax & Wild & & Endangered & Ahmad et al., 2012 \\
\hline Melia azedirach & Cultivated & & Vulnerable & Ahmad et al., 2012; Khan \& Hussain., 2013 \\
\hline Mentha arvensis & Wild & & Vulnerable & Abbas et al., 2013; Khan., 2011 \\
\hline Mentha longifolia & Wild & Endemic & Near threatened & Abbas et al., 2013; Khan., 2011; Ahmad et al.,2009 \\
\hline Mentha viridis & & & Protected & Hussain et al., 2012 \\
\hline Morus nigra & Cultivated & & Endangered & Khan \& Hussain., 2013; Ahmad et al., 2012 \\
\hline Ocimum bacilum & Cultivated & & Vulnerable & Ahmad et al., 2012; Khan \& Hussain., 2013 \\
\hline Olea ferruginia & Wild & Endemic & Endangered & Ahmad et al., 2012; Ahmad et al., 2008 \\
\hline Origanum vulgare & Wild & Endemic & Vulnerable & Abbas et al., 2013; Khan., 2011; Akhtar et al., 2013 \\
\hline Oxalis carniculata & Wild & Endemic & Protected & Ahmad., 2007; Hussain et al., 2012; Akhtar et al., 2013 \\
\hline Phyllanthus emblica & & & Endangered & Ahmad et al., 2012 \\
\hline Plectranthus rugosus & & & Vulnerable & Ahmad et al., 2012 \\
\hline Rosa indica & Wild & & Vulnerable & Ahmad., 2007; Khan \& Hussain., 2013 \\
\hline Salvadora oleoides & Wild & Endemic & Endangered & Ahmad., 2007; Khan \& Hussain., 2013; Ahmad et al., 2008 \\
\hline Thymus linearis & Wild & Endemic & Critically Endangered & Abbas et al., 2013; Khan., 2011; Akhtar et al., 2013 \\
\hline Viola serpens & Wild & & Threatned & Sher et al., 2011; Abbas et al., 2013 \\
\hline Vitex negundo & & & Vulnrable & Khan \& Hussain., 2013 \\
\hline Zanthoxylum armatum & Wild & Endemic & Critcally Endangered & Ahmad., 2007; Ahmad et al., 2012; Akhtar et al., 2013 \\
\hline
\end{tabular}




\section{References}

1. Abbas, H., Qaiser, M. and Alam, J. (2010). Conservation status of Cadaba heterotricha stocks (Capparaceae): an endangered species in Pakistan. Pak. J. Bot., 42: 35-46.

2. Abbas, Q., Qureshi, R., Naqvi, A., Khan, S. and Hussain, I. (2013). Floristic inventory and ethnobotanical study of the Naltar valley (Karakoram Range), Gilgit, Pakistan. Pak. J Bot., 45: 269-277.

3. Abbasi, A., Ahmed, M. and Zafar, M. (2010). Herbal medicines used to cure various alignments by the inhabitants of Abbottabad district, North West Fronteir Province, Pakistan. Indian J Trad Knowl., 9:175-183.

4. Abbasi, A., Khan, M., Munir, H., Shah, M., Shah, M., Pervez, A. and Ahmad, M. (2013). Ethnobotanical appraisal and cultural values of medicinally important wild edible vegetables of Lesser Himalayas-Pakistan. J Ethnobiol Ethnomed, 9:1-16.

5. Abbasi, A.M., Khan, M.A., Ahmad, M., Qureshi, R., Arshad, M., Jahan, S., Zafar, M. and Sultana, S. (2010). Ethnobotanical study of wound healing herbs among the tribal communities in northern Himalaya ranges district Abbottabad, Pakistan. Pak J Bot., 42: $3747-3753$.

6. Abbasi, H., Qaiser, M. and Naqvi, B. (2010). Rapid in vitro multiplication of Acacia nilotica subsp. Hemispherica, a critically endangered endemic taxon. Pak J Bot., 42: 4087-4093.

7. Adnan, M., Ullah, I., Tariq, A., Murad, W., Azizullah, A., Khan, A. and Ali, N. (2014). Ethnomedicine use in the war affected region of northwest Pakistan. J Ethnobiol Ethnomed, 10: 1-16.

8. Afzal, S., Afzal, N., Awan, M., Khan, T., Gilani, A., Khanum, R. and Tariq, S. (2009). Ethno-botanical studies from Northern Pakistan, J Ayub Med Coll Abbottabad. 21: 125-139.

9. Ahmad, I., Ahmad, M., Hussain, M., Hameed, M., Ashraf, Y., Saghir, M. and Koukab, S. (2009). Spatio-temporal effects on species classification of medicinal plants in Soone Valley of Pakistan. Int J Agr Biol., 11: 64-68.

10. Ahmad, K., Kayani, W., Hameed, M., Ahmad, F. and Nawaz, T. (2012). Floristic diversity and ethnobotany of Senhsa, District Kotli, Azad Jammu and Kashmir (Pakistan). Pak J Bot., 44: 195-201.

11. Ahmad, K., Khan, Z., Ashraf, M., Hussain, M., Ibrahim, M. and Valeem E. (2008). Status of plant diversity at Kufri (Soone valley) Punjab, Pakistan and prevailing threats therein. Pak J Bot., 40: 993-997.

12. Ahmad, K., Qureshi, R., Hameed, M. and Nawaz, F. (2012). Conservation assessment and medicinal importance of some plants resources from Sharda, Neelum Valley, Azad Jammu and Kashmir, Pakistan. Int J Agr Biol., 14: 997-1000.

13. Ahmad, M., Khan, M., Zafar, M. and Sultana, S. (2007). Treatment of common ailments by plant-based remedies among the people of District Attock (Punjab) of Northern Pakistan. Afr J Tradit, Complement, and Altern Med., 4:112 -120.

14. Ahmad, S. (2007). Medicinal wild plants from Lahore-Islamabad Motorway (M-2). Pak J Bot., 39: 355-375.

15. Akhtar, N., Rashid, A., Murad, W. and Bergmeier, E. (2013). Diversity and use of ethno-medicinal plants in the region of swat, north Pakistan. J Ethnobiol Ethnomed, 9: 1-16.

16. Alam, J. and Ali, S.L. (2009). Conservation status of Astraglus gilgitensis Ali (Fabaceae): a critically endangered species in Gilgit district, Pakistan. Phyton. (Horn, Austria), 48: 211-223.

17. Alamgeer, Ahmad, T., Rashid, M., Malik, M. and Mushtaq, M. (2013). Ethnomedicinal Survey of plants of Valley Alladand Dehri, Tehsil Batkhela, District Malakand, Pakistan. Int J Basic Med Sci Pharm., 3:23-32.

18. Ali, H. and Qaiser, M. (2009). The ethnobotany of Chitral valley, Pakistan with particular refrence to medicinal plants. Pak J Bot., 41: 2009-2041.

19. Al-Mofleh, I.A., Alhaider, A.A., Mossa, J.S., Al-Sohaibani, M.O., Al-Yahya, M.A., Rafatullah, S., Shaik, S.A. (2008). Gastroprotective effect of an aqueous suspension of black cumin Nigella sativa on necrotizing agents-induced gastric injury in experimental animals. Saudi J Gastroenterol., 14: 128-134.

20. Al-Mofleh, I.A., Alhaider, A.A., Mossa, J.S., Al-Sohaibani, M.O., Rafatullah, S. and Qureshi, S. (2006). Protection of gastric mucosal damage by Coriandrum sativum L. pretreatment in Wistar albino rats. Environ Toxicol Pharmacol., 22: 64-69.

21. Amiri, M. and Joharchi, M. (2013). Ethnobotanical investigation of traditional medicinal plants commercialized in the markets of Mashhad, Iran. Avicenna J Phytomed., 3: 254-271.

22. Arshad, M. and Ahmad, M. (2004). Ethnobotanical study of Galliyat for botanical demography and bioecological diversification. Ethnobotanical Leaflets, 1: 1-30.

23. Arshad, M., Ahmad, M., Ahmed, E., Saboor, A., Abbas, A. and Sadiq, S. (2014). An ethnobiological study in Kala Chitta hills of Pothwar region, Pakistan: multinomial logit specification. J Ethnobiol Ethnomed., 10: 1-17.

24. Arshad, M., Nisar, M., Majeed, A., Ismail, S. and Ahmad, M. (2011). Ethnomedicinal flora in District Sialkot, Punjab, Pakistan. MiddleEast J Sci Res, 9: 209-214.

25. Awan, M., Jamal, Z. and Khan, A. (2013). Ethno-botanical studies of economically important plants from mountaious region of GilgitBaltistan, Pakistan. Sci Technol Develop., 32: 308-318.

26. Awan, M., Shah, M., Akbar, G. and Ahmad, S. (2001). Traditional uses of economically important plants of Chitral District, Malakand division, NWFP, Pakistan. Pak J Bot., 33: 587-598.

27. Bako, S.P., Bakfur M.J., John, I. and Bala, E.I. (2005). Ethnomedicinal and phytochemical profile of some savanna plant species in Nigeria. Int J Bot., 1: 147-150.

28. Ballabh. B. and Chaurasia, O. (2009). Medicinal plants of cold desert Ladakh used in the treatment os stomach disorder. Indian J Trad Knowl., 8: 185-190.

29. Bandyopadhyay, D., Biswas, K., Bhattacharyya, M., Reiter, R.J. and Banerjee, R.K. (2001). Gastric toxicity and mucosal ulceration induced by oxygen-derived reactive species: protection by melatonin. Curr Mol Med., 1: 501-513.

30. Barkattullah, Ibrar, M. and Hussain, F. (2009). Ethnobotanical studies of plants of Charkotli Hills, Batkhela District, Malakand, Pakistan. Front Biol (Beijing), 4: 539-548.

31. Begum, S., Islam, N., Adnan, M., Tariq, A., Yasmin, A. and Hameed, R. (2014). Ethnomedicines of highly utilized plants in the temperate Himalayan region. Afr J Tradit Complem Altern Med., 11: 132-142.

32. Belayneh, A., Asfaw, Z., Demissew, S. and Bussa, N. (2012). Medicinal plants potential and use by pastoral and agro-pastoral communities in Erer Valley of Babile Wereda, Eastern Ethiopia. J Ethnobiol Ethnomed., 8: 1-11. 
33. Biswas, K.R., Khan, T., Monalisa, M., Swarna, A., Ishika, T., Rahman, M. and Rahmatullah, M. (2011). Medicinal Plants Used by Folk Medicinal Practitioners of Four Adjoining Villages of Narail and Jessore Districts, Banglades. Am.-Eurasian J Sustain Agric., 5: $23-33$.

34. Butt, A.S., Salih, M., Jafri, W., Yakoob, J., Wasay, M. and Hamid, S. (2011). Irritable Bowel Syndrome and Psychiatric Disorders in Pakistan: A Case Control Study. Hindawi, 1-6.

35. Butt, A.S., Salih, M., Jafri, W., Yakoob, J., Wasay, M. and Hamid, S. (2012). Irritable bowel syndrome and psychiatric disorders in Pakistan: A Case Control Study. Gastroenterol Res Pract., 2012: 1-6".

36. Chaudary, M., Ahmed, F., Maqbool, M. and Hussain, T. (2013). Ethnomedicinal inventory of flora of Maradori valley, District Forward Khahuta, Azad Kashmir, Pakistan. Am J Res Commun., 1: 239-261.

37. Farooq, S., Barki, A., Khan, M. and Fazal, H. (2012). Ethnobotanical studies of the flora of Tehsil Birmal in South Waziristan Agency, Pakistan. Pak J Weed Sci Res., 18: 277-291.

38. Gadekar, R., Singour, P.K., Chaurasiya, P.K., Pawar, R.S. and Patil, U.K. (2010). A potential of some medicinal plants as an antiulcer agents. Pharmacog Rev., 4: 136-146.

39. Gulshan, A., Dasti, A., Hussain, S., Atta, M. and Din, M. (2012). Indigenous uses of medicinal plants in rural areas of Dera Ghazi Khan, Punjab, Pakistan. J Agricul Bio Sci., 7: 750-762.

40. Gurav, S. and Gurav, N. (2014). A Comprehensive review: Bergenia ligulata Wall - A controversial clinical candidate. Int J Pharm Sci Rev Res., 5: 1630-1642.

41. Hamayun, M. (2007). Traditional uses of some medicinal plants of Swat valley, Pakistan. Ind J Trad Knowl., 6: 631-641.

42. Hamayun, M., Afzal, S. and Khan, M. (2006). Ethnopharmacology indigenous collection and preservation techniques of some frequently used medicinal plants of Utror and Gabral, District Swat, Pakistan. Afr J Tradit Complem Altern Med., 3: 57-73.

43. Hamayun, M., Khan, S., Sohn, E. and Lee, I. (2006). Folk medicinal knowledge and conservation status of some economically valued medicinal plants of District Swat, Pakistan. Lyonia., 11: 101-113.

44. Haq, I. (1993). Medicinal Palnts- Report of committee on Economic and Therapeutic importance of medicinal plants. Ministry of Health. Government of Pakistan.

45. Hassan, H., Murad, W., Tariq, A. and Ahmad, A. (2014). Ethnoveterinary study of medicinal plants in Malakand Valley, District Dir (Lower), Khyber Pakhtunkhwa, Pakistan. Ir Vet J., 67: 1-6.

46. Hayat, M., Khan, M., Ahmad, M., Shaheen, N., Yasmin, G. and Akhter, S. (2008). Ethnotaxonomical approach in the identification of useful medicinal flora of tehsil Pindigheb (District Attock) Pakistan. Ethnobot Res Appl., 6: 35-62.

47. Hayat, M., Khan, M., Ashraf, M. and Jabeen, S. (2009). Ethnobotany of the Genus Artemisia L. (Asteraceae) in Pakistan. Ethnobot Res Appl., 7: 147-162.

48. Hazrat, A., Nisar, M., Shah, J. and Ahmad, S. (2011). Ethnobotanical study of some elite plants belonging to Dir, Kohistan valley, Khaber Pukhtunkhwa, Pakistan. Pak J Bot., 43: 787-795.

49. Hocking, G.M. (1958). Pakistan medicinal plants. Qualitas Plantarium Ethnobotanical Material Vegetablies., 5: 145-153.

50. Hossan, M., Hanif, A., Khan, M., Bari, S., Jahan, R. and Rahmatullah, M. (2009). Ethnobotanical survey of the Tripura tribe of Bangladesh. Am.-Eurasian J Sustain Agric., 3: 253-261.

51. Husain, S., Malik, R., Javaid, M. and Bibi, S. (2008). Ethnobotanical properties and uses of medicinal plants of morgah biodiversity park, Rawalpindi. Pak J Bot., 40: 1897-1911.

52. Hussain, K., Nisar, M., Majeed, A., Khalid, Nawaz, K. and Bhatti, K. (2010). Ethnomedicinal Survey for Important Plants of Jalalpur Jattan, District Gujrat, Punjab, Pakistan. Ethnobotanical Leaflets., 14: 807-25.

53. Hussain, W., Hussain, J., Ali, R., Hussain, S., Khan, M., Khan, I., Shinwari, Z., Lopes, W. and Nascimento, I. (2012). Phytomedicinal Studies of Kurram Agency in the FederallyAdministered Tribal Areas (FATA) of Pakistan. J Appl Pharma Sci., 2: 081-085.

54. Ibrar, M., Hussain, F. and Sultan, A. (2007). Ethnobotanical studies on plant resources of Ranyal Hills, District Shangla, Pakistan. Pak J Bot., 2: 329- 337.

55. Ignacimuthu, S., Ayyanar, M. and Sivaraman, S. (2006). Ethnobotanical investigations among tribes in Madurai District of Tamil Nadu (India). J Ethnobiol Ethnomed., 11: 1-7.

56. Iqbal, H., Sher, Z. and Khan, Z. (2011). Medicinal plants from salt range Pind Dadan Khan, district Jhelum, Punjab, Pakistan. J Med Pl Res., 5: 2157-2168.

57. Jan, S., Khan, M., Din, S., Murad, W., Hussain, M. and Ghani, A. (2008). Herbal rmidies used for gastrointestinal disorders in Kaghan valley, NWFP, Pakistan. Pak J Weed Sci Res., 14: 169-200.

58. Jones, W. and Kinghorn, A. (2008). Biologically active natural products of the genus Callicarpa. Curr Bioact Compd., 4: 15-32.

59. Khan, A., Farooqui, A., Raza, Y., Rasheed, F., Manzoor, H., Akhtar, S.S., Quraishy, M.S., Rubino, S., Kazmi, S.U. and Paglietti, B. (2013). Prevalence, diversity and disease association of Helicobacter pylori in dyspeptic patients from Pakistan. J Infect Dev Ctries., 7: 220-228.

60. Khan, M. and Hussain, F. (2013). Conservation status of plant species in Tehsil Takht-e-Nasrati, District Karak, Khyber Pakhtun Khawa, Pakistan. Int J Biodivers Cons., 5: 20-26.

61. Khan, M., Khan, M. and Hussain, M. (2012). Ethno veterinary medicinal uses of plants of Poonch valley Azad Kashmir. Pak J Weed Sci Res., 18: 495-507.

62. Khan, N., Ahmed, M., Ahmed, A., Shaukat, S.S., Wahab, M., Ajaib, M., Siddiqui, M.F. and Nasir, M. (2011). Important medicinal plants of Chitral Gol National Park (Cgnp) Pakistan. Pak J Bot., 2: 797-809.

63. Khan, S., Page, S., Ahmad, H., Shaheen, H., Ullah, Z., Ahmad, M. and Harper, D. (2013). Medicinal flora and ethnoecological knowledge in the Naran Valley, Western Himalaya, Pakistan. J Ethnobiol Ethnomed., 9: 1-29.

64. Khazaei, M. and Salehi, H. (2006). Protective effect of Falcaria vulgaris extract on ethanol induced gastric ulcer in rat. Iranian J Pharmacol Therp., 5: 43-46.

65. Krishna, N., Varma, Y., and Saidulu, C. (2014). Ethnobotanical studies of Adilabad District, Andhra Pradesh, India. J Pharma Phytochem., 3: 18-36.

66. Kumar, M., Paul, Y. and Anand, V. (2009). An Ethnobotanical Study of Medicinal Plants used by the Locals in Kishtwar, Jammu and Kashmir, India. Ethnobotanical Leaflets., 13: 1240-1256.

67. Kumari, S., Daizy, R., Batish, D., Singh, H., Negi, K., and Kohli, R. (2013). An ethnobotanical survey of medicinal plants used by Gujjar Community of Trikuta Hills in Jammu and Kashmir, India. J Med P1 Res., 7: 2111-2121. 


\section{Adnan et al., Afr J Tradit Complement Altern Med. (2015) 12(5):99-117}

\section{http://dx.doi.org/10.4314/ajtcam.v12i5.15}

68. Lambert, R. (2010). Oral and Pharyngeal Cancer: World Gastrogentrology News. Official e-newsletter of the World Gastroenterology Organisation, 15(3).

69. Mahmood, A., Mahmood, A., Malik, R. and Shinwari, Z. (2013). Indigenous knowledge of medicinal plants from Gujranwala district, Pakistan. J Ethnopharmacol., 148: 714-723.

70. Mahmood, A., Malik, R., Shinwari, Z. and Mahmood, A. (2011). Ethnobotanical survey of plants from Neelum, Azad Jammu and Kashmir, Pakistan. Pak J Bot., 43: 105-110.

71. Majumdar, K. and Datta, B. (2013). Practice Pattern of Traditional Pharmaceutical Formulations by the Tribes of Tripura, Northeast India. Global J Pharmacol., 7: 442-447.

72. Manan, Z., Sirajuddin, Razzaq, A., Islam, M. and Ikramullah, (2007). Diversity of medicinal plants in Wari subdivision District Upper Dir, Pakistan. Pak J Biol Sci., 13: 21-28.

73. Mardaninejad, S., Janghorban, M. and Vazirpour, M. (2013). Collection and identification of medicinal plants used by the indigenous people of Mobarakeh (Isfahan), southwestern Iran. J Herb Drug., 4: 23-32.

74. Marwat, S., Rehman, F. and Khan, I. (2012). Tracing the Useful Ethnophytomedicinal Recipes of Angiosperms Used Against Jaundice and Hepatitis in Indo-Pak Subcontinent. World App Sci J., 18: 1243-1252.

75. Marwat, S., Rehman, F., Usman, K., Khakwani, A., Ghulam, S., Anwar, N., Sadiq, M. and Khan, S. (2011). Medico-ethnobotanical studies of edible wild fruit plants species from the flora of north western Pakistan (D. I. Khan). J Med Plants Res., 5: $3679-3686$.

76. Mitscher, L.A., Park, Y.H. and Clark, D. (1980). Antimicrobial agents from higher plants, flavonoids and related substances from Glycyrhiza glabra. J Nat Prod., 43: 259-69.

77. Mukherjee, D. (2013). Indigenous Knowledge and Practices of High Value Medicinal Plants among Nepalese community in Darjeeling Himalaya. Time J Agricul Vet Sci., 1: 1-10.

78. Murad, W., Ahmad, A., Ishaq, G., Khan, M., Khan, A., Ullah, I., Azizullah and Khan, I. (2012). Ethnobotanical studies on plants resources of Hazar Nao Forest, District Malakand, Pakistan. Pak J Weed Sci Res., 18: 509-527.

79. Murad, W., Azizullah, A., Adnan, M., Tariq, A., Khan, K., Waheed, S. and Ahmad, A. (2013). Ethnobotanical assessment of plant resources of Banda Daud Shah, District Karak, Pakistan. J Ethnobiol Ethnomed., 9: 1-10.

80. Mussarat, S., Salam, N., Tariq, A., Wazir, S., Ullah, R. and Adnan, M. (2014). Use of Ethnomedicinal Plants by the People Living around Indus River. Evidence Based Complem Alt., 2014: 1-14.

81. Mustafa, B., Hajdari, A., Krasniqi, F., Hoxha, E., Ademi, H., Quave, C. and Pieroni, A. (2012). Medical ethnobotany of the Albanian Alps in Kosovo. J Ethnobiol Ethnomed., 8: 1-14.

82. Ningombam, D., Devi1, S., Singh, P., Pinokiyo, A. and Thongam, B. (2014). Documentation and Assessment on Knowledge of EthnoMedicinal Practitioners: A Case Study on Local Meetei Healers of Manipur. Int J Pharma Biosci., 9: 53-70.

83. Noor, A., Khatoon, S. and Ahmed, M. (2012). Enumeration of the ethnobotanical uses of some herbs in Astore valley, Gilgit-Baltistan, Pakistan with particular refrence to health care purposes. Fuuast J Biol., 2: 31-48.

84. O'Ryan, M., Prado, V. and Pickering, L.K. (2005). A millennium update on pediatric diarrheal illness in the developing world. Seminars in Pediatric Infec Dis., 16: 125-136.

85. Patel, Y., Patel, R., Mahato, A. and Joshi, P. (2013). Status and diversity of ethno-medicinal plants of Dhinadhar Hill, Kachchh District, Gujarat. Int J Plant Env Sci., 3: 265-273.

86. Poffenberger, M., McGean, B., Khare, A. and Campbell, J. (1992). Community forest economy and use pattern: participatory rural apraisal method in south Gujarat, India. Field Method Manual Volume II. New Delhi, Society for promotion of Wastelands Development.

87. Prusti, A. and Behera, K. (2007). Ethnobotanical Exploration of Malkangiri District of Orissa, India. Ethnobotanical Leaflets., 11: 122140.

88. Punjani, B. (2002). Ethnobotanical aspects of some plants of Aravalli Hills in North Gujrat. Ancient Sci Life, 21: 268-280.

89. Qamar, Q., Anwar, M., Dar, N. and Ali, U. (2010). Ethno-Botanical Study of Wild Medicinal Plants of Neelum Valley, Azad Jammu and Kashmir, Pakistan. Pak J Wild., 1: 25-30.

90. Qureshi, R., Bhatti, G. and Memon, R. (2010). Ethnomedicinal uses of herbs from northern part of Nara Desert, Pakistan. Pak J Bot., 42: 839-851.

91. Qureshi, R., Ghufran, M., Sultana, K., Ashraf, M., Khan A. (2006). Ethnobotanical Studies of Medicinal Plants of Gilgit District and Surrounding Areas. Ethnobot Res Appl., 5: 115-122.

92. Qureshi, R., Gilania, S. and Ashrafb, M. (2007). Ethnobotanical studies with special reference to plants phenology at Sudhan Gali and Ganga Chotti Hills (District Bagh, A.K). Elec. J. Env. Agri. Food Chem., 6: 2207-2215.

93. Qureshi, R., Maqsood, M., Arshad, M. and Chuadary, A. (2011). Ethnomedicinal uses of plants by the people of Kadhi areas of Khushab, Punjab, Pakistan. Pak J Bot., 43: 121-133.

94. Rafatullah, S., Tariq, M., Al-Yahya, M.A., Mossa, J.S. and Ageel, A.M. (1990). Evaluation of turmeric (Curcuma longa) for gastric and duodenal antiulcer activity in rats. J Ethnopharmacol., 29: 25-34.

95. Rahmatullah, M., Mukti, I., Haque, A., Mollik, M., Parvin, K., Jahan, R., Chowdhury, M. and Rahman, T. (2009). An ethnobotanical survey and pharmacological evaluation of medicinal plants used by the Garo Tribal Community living in Netrakona district, Bangladesh. Advan Nat Appl Sci., 3: 402-418.

96. Rahmatullah, M., Haque, M., Islam, S., Jamal, F., Bashar, A., Ahmed, R., Ahmed, I., Jahan, R., Ahsan and Chowdhury, M. (2010). A Survey on the Use of Medicinal Plants by Folk Medicinal Practitioners in Three Areas of Pirojpur District, Bangladesh. Am.-Eurasian J. Sustain. Agric., 4: 247-259.

97. Rahmatullah, M., Ishika, T., Rahman, M., Swarna, A., Khan, T., Monalisa, M., Seraj, S., Mou, S., Mahal, M. and Biswas, K. (2011). Plants prescribed for both preventive and therapeutic purposes by the traditional healers of the Bede community residing by the Turag river, Dhaka District. Am.-Eurasian J. Sustain. Agric., 5: 325-331.

98. Rajasab, A. and Isaq, M. (2004). Domentation of folk knowledge on edible wild plants of North Karnata. Ind J Tradit Knowl., 3: 419429

99. Rashid, A. (2012). Medicinal plant diversity utilised in the treatment of gastrointestinal disorders by the Gujjar-Bakerwal tribe of District Rajouri of Jammu and Kashmir State Abdul. Indian Journal of Scientific Research, 3: 115-119.

100. Rauf, F., Qureshi, R. and Shaheen, H. (2012). Folk medicinal uses of indigenous plant species of Barroha, Bhara Kahu and Maanga in Islamabad, Pakistan. J Med Pl Res., 6: 2061-2070.

101. Ribeiro, H. (2000). Diarrheal disease in a developing nation. Am J Gastroenterol., 95: 14-15. 
Adnan et al., Afr J Tradit Complement Altern Med. (2015) 12(5):99-117

http://dx.doi.org/10.4314/ajtcam.v12i5.15

102. Safa, O., Soltanipoor, M., Rastegar, S., Kazemi, M., Dehkordi, K. and Ghannadi, A. (2012). An ethnobotanical survey on hormozgan province, Iran. Avicenna J Phytomed., 3: 64-81.

103. Sandhya, B., Thomas, S., Isabel, W. and Shenbagarathai, R. (2006). Ethnomedicinal plants used by the valaiyan community of Piranmalai hills (reserved forest), Tamilnadu, India. - A Pilot Study. Afr J Trad Complem Altern Med., 3: 101-114.

104. Shadangi, A., Panda, R. and Patra, A. (2012). Ethnobotanical studies of wild flora at G. Udayagiri forest in eastern Ghat, Odisha. J Environ Sci Toxicol Food Technol., 2: 25-37.

105. Shah, G. and Khan, M. (2006). Common Medicinal Folk Recipes of Siran Valley, Mansehra, Pakistan. Ethnobotanical Leaflets., 10: 4962.

106. Shaheen, H. and Shinwari, Z. (2012). Phytodiversity and endemicrichness of Karambar lake vegetation from Chitral, HindukushHimalayas. Pak J Bot., 44: 15-20.

107. Shaheen, H., Rahmatullah, Qureshi, R., Akram, A. and Gulfraz, M. (2012). Some important medicinal flora of Noorpur Thal, Khushab, Pakistan. Arch Sci., 65: 57-73.

108. Sharma, P., Thakur, S., Manuja, S., Rana, R., Kumar, P., Sharma, S., Chand, J., Singh, A. and Katoch, K. (2011). Observations on Traditional Phytotherapy among the Inhabitants of Lahaul Valley through Amchi System of Medicine-A Cold Desert Area of Himachal Pradesh in North Western Himalayas, India. Sci Res., 2: 93-102.

109. Sheikh, K., Ahmad, T. and Khan, M.A. (2002). Use, exploitation and prospects for conservation: people and plant biodiversity of Naltar Valley, northwestern Karakorums, Pakistan. Biodivers Cons., 11: 715-742.

110. Sher, H. and Hussain, F. (2009). Ethnobotanical evaluation of some plant resources in Northern part of Pakistan. Afr J biotechnol. 8: 4066-4076

111. Sher, Z., Khan, Z. and Hussain, F. (2011). Ethnobotanical studies of some plants of Chagharzai valley, District Buner, Pakistan. Pak J Bot., 43: 1445-1452.

112. Shinwari, Z.K., Gilani, S.S. and Akhlas, M. (2003). Sustainable Harvest of Medicinal Plants at Barand Shinaki Valleys, Gilgit (Northern Pakistan). W WF - P, Gilgit: Consultancy Report.

113. Shinwari, Z.K., Salima, M., Faisal, R., Huda, S. and Asrar, M. (2013). Biological screening of indigenous knowledge based plants used in diarrheal treatment. Pak J Bot., 45: 1375-1382.

114. Singh, A., Kumar, A. and Tewari, D. (2012). An ethnobotanical survey of medicinal plants used in Terai forest of western Nepal. J Ethnobiol Ethnomed., 37: 1-14

115. Sultana, S., Khan, M., Ahmad, M. and Zafar, M. (2006). Indigenous Knowledge of Folk Herbal Medicines by the Women of District Chakwal, Pakistan. Ethnobotanical Leaflets. 10: 243-253.

116. Tally, N.G., Stanghellini, V., Heading, R.C., Koch, K.L., Malagelada, J.R. and Tytgat, G.N. (1999). Functional gastrointestinal disorder. Gut., 45: 1137-1142.

117. Tareen, R., Bibi, T., Khan, M., Ahmad, M. and Zafar, M. (2010). Indigenous knowlege of folk medicine by the women of Kalat and Khuzdar regions of Balochistan, Pakistan. Pak J Bot., 42: 1465-1485.

118. Tarnawski, A.S. (2005). Cellular and molecular mechanisms of gastrointestinal ulcer healing. Dig Dis Sci., 50: 24-33.

119. Thapar, N. and Sanderson, I.R. (2004). Diarrhea in children: an interface between developing and developed countries. The Lance, 363: 641-653.

120. Ullah, R., Hussain, Z., Iqbal, Z., Hussain, J., Khan, F., Khan, N., Muhammad, Z., Ayaz, S., Ahmad, S., Rehman, N. and Hussain, I. (2010). Traditional uses of medicinal plants in Darra Adam Khel NWFP Pakistan. J Med Plants Res., 4: 1815-1821.

121. Walter, W. and Hamilton, A. (1993). The vital wealth of plants. UK: Bates and Sons Ltd.

122. Wariss, H., Ahmad, S., Anjum, S. and Alam, K. (2014). Ethnobotanical studies of dicotyledonous plants of Lal Suhanra National Park, Bahawalpur, Pakistan. Int J Sci Res., 3: 2452-2460.

123. Wazir, S., Dasti, A. and Shah, J. (2004). Common medicinal plants of Chapursan valley, Gojal II, Gilgit-Pakistan. J Res (Science)., 15: 41-43.

124. Yuan, Y., Padol, I.T. and Hunt, R.H. (2006). Peptic ulcer disease today. Nat. Rev. Gastroenterol. Hepatol. 3: 80- 89.

125. Zaidi, F.H., Yamada, K., Kadowaki, M., Usmanghani, K. and Sugiyama, T. (2009). Bactericidal activity of medicinal plants, employed for the treatment of gastrointestinal ailments, against Helicobacter pylori. J Ethnopharmacol. 121: 286-291. 